\title{
A nonlinear $p$-Laplace equation with critical Sobolev-Hardy exponents and Robin boundary conditions
}

Lingyu Jin ${ }^{*}$ and Lang Li

\section{"Correspondence:}

jinlingyu@scau.edu.cn Department of Applied

Mathematics, South China

Agricultural University, Guangzhou,

510642, P.R. China

\begin{abstract}
In this paper, we are concerned with a nonlinear $p$-Laplace equation with critical Sobolev-Hardy exponents and Robin boundary conditions. Through a compactness analysis of the functional corresponding to the problem, we obtain the existence of positive solutions for this problem under different assumptions.
\end{abstract}

MSC: 35J10; 35J20; 35J60

Keywords: compactness; positive solution; Sobolev-Hardy exponent

\section{Introduction}

We are concerned with the following class of boundary value problems:

$$
\begin{cases}-\Delta_{p} u-\mu \frac{|u|^{p-2} u}{|x|^{p}}+\lambda|u|^{p-2} u=\frac{|u|^{*}(s)-2}{|x|^{s}}+\eta|u|^{q-2} u, & \text { in } \Omega, \\ |\nabla u|^{p-1} \frac{\partial u}{\partial v}+\alpha(x)|u|^{p-2} u=0, & \text { on } \partial \Omega\end{cases}
$$

where $0 \in \bar{\Omega} \subset \mathbb{R}^{n}, 2 \leq p<n, p^{*}(s)=p(n-s) /(n-p), p<q<p^{*}(s), 0 \leq s<p, \mu<\bar{\mu}:=\frac{(n-p)^{p}}{p^{p}}$, $\eta \geq 0$ and $\lambda \in \mathbb{R}^{1}$ are parameters, $\alpha(x) \in C(\partial \Omega), \alpha(x) \geq 0 . \Omega$ is a bounded domain with a smooth $C^{2}$ boundary, $v$ denotes the unit outward normal to $\partial \Omega$.

The main interest of this kind of problems is the presence of the singular potential $\frac{1}{|x|^{s}}$, $0 \leq s \leq p, \frac{1}{|x|^{s}}$ relating to the Hardy inequality. In the special case when $\mu=0$, problem (1.1) is related to the well-known Sobolev-Hardy inequality

$$
\left(\int_{\Omega} \frac{u^{q}}{|x|^{s}} d x\right)^{\frac{p}{q}} \leq \frac{1}{C_{q, s, p}} \int_{\Omega}|\nabla u|^{p} d x, \quad \forall u \in W_{0}^{1, p}(\Omega),
$$

which is essentially due to Caffarelli, Kohn and Nirenberg (see [1]), where $1<p<n, q \leq$ $p^{*}(s), C_{q, s, p}$ is a positive constant depending on $p, q$, $s$. When $q=s=p$, the above Sobolev inequality becomes the well-known Hardy inequality (see [1, 2])

$$
\int_{\Omega} \frac{|u|^{p}}{|x|^{p}} d x \leq \frac{1}{\bar{\mu}} \int_{\Omega}|\nabla u|^{p} d x, \quad \forall u \in W_{0}^{1, p}(\Omega) .
$$

(c) $2015 \mathrm{Jin}$ and Li. This article is distributed under the terms of the Creative Commons Attribution 4.0 International License (http://creativecommons.org/licenses/by/4.0/), which permits unrestricted use, distribution, and reproduction in any medium, provided you give appropriate credit to the original author(s) and the source, provide a link to the Creative Commons license, and indicate if changes were made. 
Moreover, the constant $\bar{\mu}$ is optimal and is not achieved since the Sobolev embedding is not compact even locally in any neighborhood of zero. In addition to the inverse potential, there is the presence of the critical Sobolev exponents and critical Sobolev-Hardy exponents, which causes of loss of compactness of the embeddings. This loss of compactness leads to many interesting existence and nonexistence phenomena for the elliptic equations with critical Hardy terms (see, for example, [3-8] and the references therein).

For second-order semilinear elliptic differential equations on bounded domains, Brezis and Lieb [9] obtained an existence result of solutions for a class of elliptic equations with critical Sobolev nonlinearities by verifying a sub-level which satisfies the Palais-Smale conditions. A global compact result for a semilinear elliptic problem with critical Sobolev nonlinearities on bounded domains was obtained by Struwe [10]. Pierrotti and Terracini [11] studied a class of critical elliptic equations with Neumann boundary conditions through a compact analysis. Cao and Peng [4] got a global compact result for (1.1) (when $p=2, s=0$ ) with Dirichlet boundary conditions and showed some new blow-up phenomena. Deng, Jin and Peng [12] got a similar result for the Robin boundary problem of equation (1.1) (when $p=2, s=0$ ). In [13], with the Dirichlet boundary conditions of equation (1.1) (when $s \neq 0$ ), they got the global compact result on the whole space and a bounded smooth domain, respectively. For the elliptic differential equations on unbounded domains, there have also been some global compact results (refer to $[8,14,15])$. In this paper, we discuss a general Robin boundary problem involving critical Hardy terms and critical Sobolev-Hardy terms with $p \geq 2,0 \leq s<p$. The different assumptions on the parameter $s$ induce completely different results corresponding to the noncompactness analysis. In addition, the boundary conditions make great influence on our noncompact analysis. Not only does it change the form of our limiting equations, but it also adds more limiting equations which induce new blow-up bubble such as $D_{\mu}$ (see Corollary 1.1) to occur.

The first goal of this paper is a careful analysis of the features of a Palais-Smale sequence for the corresponding variational functional $F_{\mu}(u)$ of (1.1). To this aim, following the same idea adopted by Struwe [10] and the main techniques of [11], we shall employ the blowup technique to characterize all the energy levels where the Palais-Smale condition fails. More precisely, we shall represent any diverging Palais-Smale sequence as the sum of critical points of a family of limiting functionals, which are invariant under scaling. In our problem, due to the Hardy potential, critical Sobolev-Hardy terms, there are some critical points of a new family of limiting functionals. As a by-product, we shall find the smallest level where the Palais-Smale condition may fail. Thus we shall be able to determine safe sublevels where standard critical point theorems can be applied. The second purpose of this paper is to obtain the existence of critical points for the variational functional of (1.1) under different conditions by applying the previous compactness analysis.

To mention our main results, it is convenient to introduce some notations.

Firstly, we denote by $F_{\mu}$ the functional associated to (1.1):

$$
\begin{aligned}
F_{\mu}(u)= & \frac{1}{p} \int_{\Omega}\left(|\nabla u|^{p}-\mu \frac{|u|^{p}}{|x|^{p}}\right) d x+\frac{1}{p} \int_{\partial \Omega} \alpha(x)|u|^{p} d \sigma-\frac{1}{p^{*}(s)} \int_{\Omega} \frac{|u|^{p^{*}(s)}}{|x|^{s}} d x \\
& +\frac{\lambda}{p} \int_{\Omega}|u|^{p} d x-\frac{\eta}{q} \int_{\Omega}|u|^{q} d x, \quad u \in W^{1, p}(\Omega) .
\end{aligned}
$$


We denote by $\lambda_{1}$ the smallest positive eigenvalue such that the following problem has a positive solution:

$$
\begin{cases}-\Delta_{p} u-\mu \frac{|u|^{p-2} u}{|x|^{p}}=\lambda|u|^{p-2} u, & x \in \Omega \\ |\nabla u|^{p-1} \frac{\partial u}{\partial v}+\alpha(x)|u|^{p-2} u=0, & x \in \partial \Omega \\ u \in W^{1, p}(\Omega) & \end{cases}
$$

i.e.,

$$
\lambda_{1}=\inf \left\{\int_{\Omega}\left(|\nabla u|^{p}-\mu \frac{|u|^{p}}{|x|^{p}}\right) d x+\int_{\partial \Omega} \alpha(x)|u|^{p} d \sigma ; \int_{\Omega}|u|^{p} d x=1, u \in W^{1, p}(\Omega)\right\} .
$$

From Lemma A.1 in the Appendix, $\lambda_{1}$ can be attained. If $\mu \leq 0$, obviously $\lambda_{1}>0$. If $\mu \in(0, \bar{\mu})$, by Lemma A.2 in the Appendix of this paper, we have

$$
\mu \int_{\Omega} \frac{|u|^{p}}{|x|^{p}} d x \leq \int_{\Omega}|\nabla u|^{p}+c(\varepsilon, \mu) \int_{\Omega}|u|^{p} d x
$$

for $u \in W^{1, p}(\Omega)$. Hence, for suitably large $\lambda>0$, we have $\lambda+\lambda_{1}>0$ for $\mu \in(-\infty, \bar{\mu})$. Now, for $\lambda>-\lambda_{1}$, we define the following norm:

$$
\|u\|=\left[\int_{\Omega}\left(|\nabla u|^{p}-\mu \frac{|u|^{p}}{|x|^{p}}+\lambda|u|^{p}\right) d x+\int_{\partial \Omega} \alpha(x)|u|^{p} d \sigma\right]^{\frac{1}{p}} .
$$

Then, by Lemma A.3 in the Appendix of this paper, $\|\cdot\|$ is equivalent to the usual norm $\|\cdot\|_{W^{1, p}(\Omega)}$.

Secondly, we denote $\mathbb{R}_{+}^{n}:=\left\{y=\left(y_{1}, y_{2}, \ldots, y_{n-1}, y_{n}\right):=\left(y^{\prime}, y_{n}\right) \in \mathbb{R}^{n} \mid y_{n}>0\right\}$ with boundary $\mathbb{R}^{n-1}=\left\{y \mid\left(y^{\prime}, 0\right) \in \mathbb{R}^{n}\right\}$. Denote $C_{0}^{\infty}(\Omega)=\left\{u \in C^{\infty}\left(\mathbb{R}^{n}\right) \mid \operatorname{supp} u \subset \subset \Omega\right\}$. The space $D^{1, p}(\Omega)$ is the completion of $C_{0}^{\infty}(\Omega)$ with respect to the norm

$$
\|u\|_{D^{1, p}(\Omega)}=\left(\int_{\Omega}|\nabla u|^{p} d x\right)^{1 / p}
$$

the space $D^{1, p}\left(\mathbb{R}_{+}^{n}\right)$ is the space of the restrictions to $\mathbb{R}_{+}^{n}$ of elements of $D^{1, p}\left(\mathbb{R}^{n}\right)$. Recall $p^{*}(s)=p(n-s) /(n-p)$ and denote $p^{*}=p^{*}(0)=\frac{n p}{n-p}$. In the following $C$ and $c$ denote various generic positive constants. $O(\varepsilon)$ denotes a quantity satisfying $|O(\varepsilon)| / \varepsilon \leq C, o(\varepsilon)$ means $|o(\varepsilon)| / \varepsilon \rightarrow 0$ as $\varepsilon \rightarrow 0$ and $o(1)$ is a generic infinitesimal value.

Finally we give the definition of the Palais-Smale sequence as follows: let $X$ be a Banach space, $\phi \in C^{1}(X, \mathbb{R})$ and $c \in \mathbb{R}$. The sequence $u_{m} \in X$ is called a Palais-Smale sequence of $\phi$ at a level $c$ if

$$
\phi\left(u_{m}\right) \rightarrow c, \quad \phi^{\prime}\left(u_{m}\right) \rightarrow 0 \quad \text { as } m \rightarrow \infty .
$$

Define

$$
S_{\mu, s}=\inf _{u \in D^{1, p}\left(\mathbb{R}^{n}\right) \backslash\{0\}} \frac{\int_{\mathbb{R}^{n}}\left(|\nabla u|^{p}-\mu \frac{|u|^{p}}{|x|^{p}}\right) d x}{\left(\int_{\mathbb{R}^{n}} \frac{|u|^{*}(s)}{|x|^{s}} d x\right)^{p / p^{*}(s)}},
$$


which plays an important role in our argument. In particular we denote $S=S_{0,0}$ and $S_{\mu}=$ $S_{\mu, 0}$.

In order to establish the global compactness result for problem (1.1), it is also convenient to introduce the problems at infinity corresponding to (1.1) as follows.

$$
\begin{aligned}
& -\Delta_{p} v=|v|^{p^{*}-2} v, \quad v \in D^{1, p}\left(\mathbb{R}^{n}\right) ; \\
& -\Delta_{p} v-\mu \frac{|v|^{p-2} v}{|x|^{p}}=\frac{|v|^{p^{*}(s)-2} v}{|x|^{s}}, \quad v \in D^{1, p}\left(\mathbb{R}^{n}\right) ; \\
& \begin{cases}-\Delta_{p} v=|v|^{p^{*}-2} v, & v \in D^{1, p}\left(\mathbb{R}_{+}^{n}\right), \\
|\nabla v|^{p-2} \frac{\partial v}{\partial v}=0, & \text { on } \mathbb{R}^{n-1} ;\end{cases} \\
& \begin{cases}-\Delta_{p} v-\mu \frac{|\nu|^{p-2} v}{\left.|x|\right|^{p}}=\frac{|v|^{p^{*}(s)-2} v}{|x|^{s}}, & v \in D^{1, p}\left(\mathbb{R}_{+}^{n}\right) \\
|\nabla v|^{p-2} \frac{\partial v}{\partial \nu}=0, & \text { on } \mathbb{R}^{n-1}\end{cases}
\end{aligned}
$$

In fact, through scaling and transforming technique, and taking the limit, the Palais-Smale sequence of (1.1) can be represent by the solutions of problems (1.5)-(1.8) (refer to Theorem 1.1).

All positive solutions of (1.5) are the well-known $(n+1)$-parameter family of

$$
U^{\varepsilon, y}(x):=\varepsilon^{(p-n) / p} U_{0}\left(\frac{x-y}{\varepsilon}\right),
$$

where

$$
U_{0}(x):=c(n)\left(1+|x|^{\frac{p}{p-1}}\right)^{\frac{p-n}{p}}
$$

for some appropriate constant $c(n)>0$. These solutions are also known to minimize the Sobolev quotient $S$, as was shown by Aubin [16]. Since $U_{0}(x)$ is radical symmetric, then

$$
\left.\frac{\partial U_{0}}{\partial v}\right|_{x_{n}=0}=-\left.\frac{\partial U_{0}}{\partial x_{n}}\right|_{x_{n}=0}=-\left.U_{0}^{\prime}(|x|) \frac{x_{n}}{|x|}\right|_{x_{n}=0}=0,
$$

which means that $U_{0}(x)$ is also the solution of (1.7).

For $0<\mu<\bar{\mu}$ and $p>s \geq 0$, Kang in [17] showed the existence of the positive solutions of (1.6), and the form of the solutions $V_{\mu}^{\varepsilon}(|x|):=\varepsilon^{\frac{p-n}{p}} V_{\mu}(|x| / \varepsilon)$, where $V_{\mu}(x)$ is the unique positive radial function in $D^{1, p}\left(\mathbb{R}^{n}\right)$ which achieves $S_{\mu, s}$. Moreover,

$$
\begin{aligned}
& V_{\mu}^{\varepsilon}(1)=\left(\frac{(n-s)(\bar{\mu}-\mu)}{n-p}\right)^{\frac{1}{p^{*}(s)-p}}, \\
& \lim _{r \rightarrow 0} r^{a(\mu)} V_{\mu}(r)=c_{1}>0, \\
& \lim _{r \rightarrow+\infty} r^{b(\mu)} V_{\mu}(r)=c_{2}>0,
\end{aligned}
$$

where $r=|x|, c_{1}$ and $c_{2}$ are constants depended on $p, n . a(\mu)$ and $b(\mu)$ are solutions of

$$
0=(p-1) \tau^{p}-(n-p) \tau^{p-1}+\mu,
$$


where $\tau \geq 0,0 \leq \mu \leq \bar{\mu}, 0 \leq a(\mu)<\frac{n-p}{p}<b(\mu)<\frac{n-p}{p-1}$. Of course, $V_{\mu}^{\varepsilon}(|x|)$ are also the solutions of (1.8).

For convenience, we also define the following quantities which will represent the amount of the functional $F_{\mu}(u)$ carried over by blowing-up bubbles:

$$
\begin{aligned}
D_{0} & :=\int_{\mathbb{R}^{n}}\left(\frac{1}{p}\left|\nabla U_{0}\right|^{p}-\frac{1}{p^{*}} U_{0}^{p^{*}}\right) d x=\frac{1}{n} S^{n / p}, \\
D_{\mu} & :=\int_{\mathbb{R}^{n}}\left(\frac{1}{p}\left|\nabla V_{\mu}\right|^{p}-\mu \frac{V_{\mu}^{p}}{|x|^{p}}-\frac{1}{p^{*}(s)} \frac{V_{\mu}^{p^{*}(s)}}{|x|^{s}}\right) d x=\frac{p-s}{(n-s) p} S_{\mu, s}^{\frac{n-s}{p-s}} .
\end{aligned}
$$

In order to unify the notations, we shall refer to the solutions of problems (1.5)-(1.8) as critical points of the following family of functionals:

$$
\begin{aligned}
& F^{\infty}(u)=\frac{1}{p} \int_{\mathbb{R}^{n}}|\nabla u|^{p} d x-\frac{1}{p^{*}} \int_{\mathbb{R}^{n}}|u|^{p^{*}} d x, \\
& F_{\mu}^{\infty}(u)=\frac{1}{p} \int_{\mathbb{R}^{n}}\left(|\nabla u|^{p}-\mu \frac{|u|^{p}}{|x|^{p}}\right) d x-\frac{1}{p^{*}(s)} \int_{\mathbb{R}^{n}} \frac{|u|^{p^{*}(s)}}{|x|^{s}} d x, \\
& F_{+}^{\infty}(u)=\frac{1}{p} \int_{\mathbb{R}_{+}^{n}}|\nabla u|^{p} d x-\frac{1}{p^{*}} \int_{\mathbb{R}_{+}^{n}}|u|^{p^{*}} d x, \\
& F_{\mu,+}^{\infty}(u)=\frac{1}{p} \int_{\mathbb{R}_{+}^{n}}\left(|\nabla u|^{p}-\mu \frac{|u|^{p}}{|x|^{p}}\right) d x-\frac{1}{p^{*}(s)} \int_{\mathbb{R}_{+}^{n}} \frac{|u|^{p^{*}(s)}}{|x|^{s}} d x .
\end{aligned}
$$

We shall prove that any diverging Palais-Smale sequence corresponding to (1.1) can be represented as sums of scaled critical points of the functionals $F_{\mu}^{\infty}(u), F_{\mu,+}^{\infty}(u)$ or $F^{\infty}(u)$, $F_{+}^{\infty}(u)$ by exploiting suitable blow-up arguments.

The first result of this paper is the following global compactness theorem.

Theorem 1.1 Let $\left\{u_{m}\right\} \subset W^{1, p}(\Omega)$ be a Palais-Smale sequence of $F_{\mu}(u)$ at level $d>0$, $u_{0}$ is a critical point of $F_{\mu}(u)$,

$$
\zeta(s)= \begin{cases}1 & \text { if } s=0 \\ 0 & \text { if } s \neq 0\end{cases}
$$

Then there exist $k_{1}, k_{2}, k_{3} \in \mathbb{N} \cup\{0\}$ such that

(i) $u_{m}$ can be decomposed as

$$
u_{m}=u_{0}+\sum_{j=1}^{k_{1}} r_{m, j}^{\frac{n-p}{p}} U_{j}\left(r_{m, j} x\right)+\zeta(s) \sum_{j=k_{1}+1}^{k_{1}+k_{2}+k_{3}} r_{m, j}^{\frac{n-p}{p}} U_{j}\left(r_{m, j}\left(x-x_{m, j}\right)\right)+\omega_{m}
$$

where $\omega_{m} \rightarrow 0$ in $W^{1, p}(\Omega)$ as $m \rightarrow+\infty$, and

$$
\begin{aligned}
\text { for } j=1, \ldots, k_{1}, r_{m, j} \rightarrow+\infty \text { as } m \rightarrow+\infty, \\
\begin{cases}U_{j} \text { satisfy }(1.6) & \text { if } 0 \in \Omega, \\
U_{j} \text { satisfy }(1.8) & \text { if } 0 \in \partial \Omega ;\end{cases}
\end{aligned}
$$




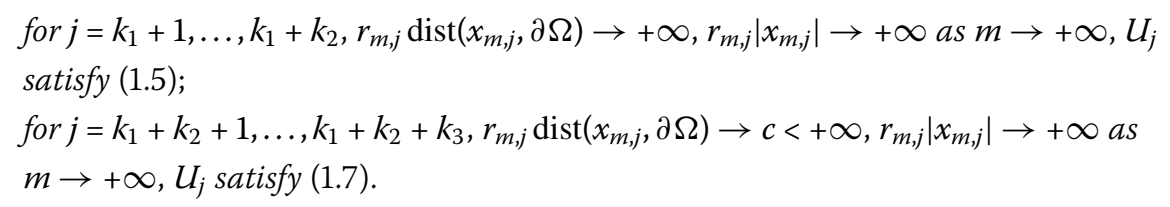

(ii) $F_{\mu}\left(u_{m}\right)$ can be decomposed as the following:

- for the case that $0 \in \partial \Omega$, as $m \rightarrow+\infty$,

$$
F_{\mu}\left(u_{m}\right)=F_{\mu}\left(u_{0}\right)+\sum_{j=1}^{k_{1}} F_{\mu,+}^{\infty}\left(U_{j}\right)+\zeta(s) \sum_{j=k_{1}+1}^{k_{1}+k_{2}} F^{\infty}\left(U_{j}\right)+\zeta(s) \sum_{j=k_{1}+k_{2}+1}^{k_{1}+k_{2}+k_{3}} F_{+}^{\infty}\left(U_{j}\right)+o(1)
$$

where

$$
\begin{aligned}
& \text { for } j=1, \ldots, k_{1}, U_{j} \text { is a solution of }(1.8) ; \\
& \text { for } j=k_{1}+1, \ldots, k_{1}+k_{2}, U_{j} \text { is a solution of }(1.5) ; \\
& \text { for } j=k_{1}+k_{2}+1, \ldots, k_{1}+k_{2}+k_{3}, U_{j} \text { is a solution of }(1.7) ;
\end{aligned}
$$

- for the case that $0 \in \Omega$, as $m \rightarrow+\infty$,

$$
F_{\mu}\left(u_{m}\right)=F_{\mu}\left(u_{0}\right)+\sum_{j=1}^{k_{1}} F_{\mu}^{\infty}\left(U_{j}\right)+\zeta(s) \sum_{j=k_{1}+1}^{k_{1}+k_{2}} F^{\infty}\left(U_{j}\right)+\zeta(s) \sum_{j=k_{1}+k_{2}+1}^{k_{1}+k_{2}+k_{3}} F_{+}^{\infty}\left(U_{j}\right)+o(1)
$$

where

$$
\begin{aligned}
& \text { for } j=1, \ldots, k_{1}, U_{j} \text { is a solution of }(1.6) ; \\
& \text { for } j=k_{1}+1, \ldots, k_{1}+k_{2}, U_{j} \text { is a solution of }(1.5) ; \\
& \text { for } j=k_{1}+k_{2}+1, \ldots, k_{1}+k_{2}+k_{3}, U_{j} \text { is a solution of }(1.7) \text {. }
\end{aligned}
$$

Corollary 1.1 Any positive Palais-Smale sequence for $F_{\mu}(u)$ at a level $d$ which is not of the form $k_{1} D_{\mu}+k_{2} D_{0}+\frac{1}{2} k_{3} D_{0}$ if $0 \in \Omega$ and the form $\frac{k_{1}}{2} D_{\mu}+k_{2} D_{0}+\frac{1}{2} k_{3} D_{0}$ if $0 \in \partial \Omega$ for $k_{1}, k_{2}, k_{3} \in \mathbb{N} \cup\{0\}$, gives rise to a nontrivial weak solution of equation (1.1).

By applying Theorem 1.1 and the mountain pass theorem [18], we can obtain the following existence theorems by proving that $F_{\mu}(u)$ satisfies the geometrical assumptions of the mountain pass theorem and that the mountain pass level is actually below the compactness threshold quoted in Theorem 1.1.

Theorem 1.2 Suppose $0 \in \Omega, p>s>0, \lambda>-\lambda_{1}, 0<\mu<\bar{\mu}$, then problem (1.1) has a positive solution if

$$
\max \left\{p, \frac{n}{b(\mu)}, \frac{p(2 n-b(\mu) p-p)}{n-p}\right\}<q<p^{*}(s)
$$

Theorem 1.3 Suppose $0 \in \Omega, s=0, \lambda>-\lambda_{1}$. Then there exists a constant $\mu^{*} \in(0, \bar{\mu})$ such that

(1) problem (1.1) has a positive solution if $0<\mu \leq \mu^{*}$; 
(2) problem (1.1) has a positive solution if

$$
\mu^{*}<\mu<\bar{\mu} \quad \text { and } \quad \max \left\{p, \frac{n}{b(\mu)}, \frac{p(2 n-b(\mu) p-p)}{n-p}\right\}<q<p^{*} .
$$

Furthermore, $\mu^{*}$ can be calculated by solving $S^{\frac{n}{p}}=2 S_{\mu}^{\frac{n}{p}}$.

Remark 1.1 For the case that $0 \in \partial \Omega$, we cannot obtain the existence of the solutions of problem (1.1) since we do not know the explicit form of the attaining functions of $S_{\mu, s}$.

This paper is organized as follows. In Section 2, we prove Theorem 1.1 by carefully analyzing the features of a Palais-Smale sequence for $F_{\mu}(u)$. In Section 3, we apply Theorem 1.1 and the mountain pass theorem [18] to obtain the existence of critical points for $F_{\mu}(u)$ under different assumptions on the parameters $\mu, \lambda$ and the fact that $0 \in \Omega$. Finally, we put some preliminaries in the last section as an appendix.

\section{Proof of Theorem 1.1}

In this section, the features of a Palais-Smale sequence for $F_{\mu}(u)$ will be analyzed by the blow-up technique adopted by Struwe [10] for the Dirichlet problem. To this end, we need the following lemma.

Lemma 2.1 Let $\left\{v_{m}\right\}_{m}$ be a Palais-Smale sequence of $F_{\mu}(u)$ at level $d>0$, and assume that $\left\{v_{m}\right\}_{m}$ converges weakly but not strongly to zero in $W^{1, p}(\Omega)$.

(1) For the case $s \neq 0$,

- if $0 \in \Omega$, there exists a positive sequence $k_{m}$ such that, up to a subsequence,

$$
w_{m}=v_{m}(x)-k_{m}^{\frac{n-p}{p}} v_{0}\left(k_{m} x\right), \quad x \in \bar{\Omega}
$$

is a Palais-Smale sequence for $F_{\mu}(u)$ in $W^{1, p}(\Omega)$ at level $d-\frac{p-s}{(n-s) p} S_{\mu, s}^{\frac{n-s}{p-s}}$, and $v_{0}$ solves (1.6). Moreover, $w_{m} \rightarrow 0$ weakly in $W^{1, p}(\Omega)$ as $m \rightarrow+\infty$;

- if $0 \in \partial \Omega$, there exists a positive sequence $k_{m}$ such that, up to a subsequence,

$$
w_{m}=v_{m}(x)-k_{m}^{\frac{n-p}{p}} v_{0}\left(k_{m} x\right), \quad x \in \bar{\Omega}
$$

is a Palais-Smale sequence for $F_{\mu}(u)$ in $W^{1, p}(\Omega)$ at level $d-\frac{p-s}{2(n-s) p} S_{\mu, s}^{\frac{n-s}{p-s}}$, and $v_{0}$ solves

(1.8). Moreover, $w_{m} \rightarrow 0$ weakly in $W^{1, p}(\Omega)$ as $m \rightarrow+\infty$.

(2) For the case that $s=0$, then either

- if $0 \in \Omega$, there exists a positive sequence $k_{m}$ such that, up to a subsequence,

$$
w_{m}=v_{m}(x)-k_{m}^{\frac{n-p}{p}} v_{0}\left(k_{m} x\right), \quad x \in \bar{\Omega}
$$

is a Palais-Smale sequence for $F_{\mu}(u)$ in $W^{1, p}(\Omega)$ at level $d-\frac{1}{n} S_{\mu}^{\frac{n}{p}}$, and $v_{0}$ solves (1.6). Moreover, $w_{m} \rightarrow 0$ weakly in $W^{1, p}(\Omega)$ as $m \rightarrow+\infty$;

- if $0 \in \partial \Omega$, there exists a positive sequence $k_{m}$ such that, up to a subsequence,

$$
w_{m}=v_{m}(x)-k_{m}^{\frac{n-p}{p}} v_{0}\left(k_{m} x\right), \quad x \in \bar{\Omega}
$$


is a Palais-Smale sequence for $F_{\mu}(u)$ in $W^{1, p}(\Omega)$ at level $d-\frac{1}{2 n} S_{\mu}^{\frac{n}{p}}$, and $v_{0}$ solves (1.8). Moreover, $w_{m} \rightarrow 0$ weakly in $W^{1, p}(\Omega)$ as $m \rightarrow+\infty$;

or there exist sequences $y_{m} \in \bar{\Omega}, K_{m} \in \mathbb{R}^{+}$such that, up to a subsequence,

Case 1:

$$
w_{m}(x)=v_{m}(x)-K_{m}^{\frac{n-p}{p}} v_{0}\left(K_{m}\left(x-y_{m}\right)\right), \quad x \in \bar{\Omega},
$$

is a Palais-Smale sequence for $F_{\mu}(u)$ at level $d-\frac{1}{2 n} S^{\frac{n}{p}}$ if $\lim _{m \rightarrow+\infty} K_{m} \operatorname{dist}\left(y_{m}, \partial \Omega\right)<+\infty$. Moreover, $w_{m} \rightarrow 0$ weakly in $W^{1, p}(\Omega)$ as $m \rightarrow+\infty$ and $v_{0}$ is the solution of $(1.7)$;

Case 2:

$$
w_{m}(x)=v_{m}(x)-K_{m}^{\frac{n-p}{p}} v_{0}\left(K_{m}\left(x-y_{m}\right)\right), \quad x \in \bar{\Omega},
$$

is a Palais-Smale sequence for $F_{\mu}(u)$ at level $d-\frac{1}{n} S^{\frac{n}{p}}$ if $\lim _{m \rightarrow+\infty} K_{m} \operatorname{dist}\left(y_{m}, \partial \Omega\right)=+\infty$. Moreover, $w_{m} \rightarrow 0$ weakly in $W^{1, p}(\Omega)$ and $v_{0}$ is the solution of (1.5).

Proof We only prove the case when $0 \leq \mu<\bar{\mu}$ since the proof of the case when $\mu<0$ is similar. By Lemma A.4 in the Appendix, we deduce that there are positive constants $c_{i}$ $(i=1,2)$ such that

$$
c_{1} \leq \int_{\Omega}\left|\nabla v_{m}\right|^{p} d x \leq c_{2}, \quad \forall m \in \mathbb{N} .
$$

From (2.7), let $\delta>0$ be small (will be determined later) such that

$$
\limsup _{m \rightarrow+\infty} \int_{\Omega}\left|\nabla v_{m}\right|^{p} d x>\delta
$$

Fix $m$, by the integral absolute continuity, $\forall \varepsilon>0$, there exists a constant $a>0$ for any set $E \subset \Omega$ and the measure $m(E)<a$, then

$$
\int_{E}\left|\nabla v_{m}\right|^{p} d x<\varepsilon
$$

Define $F(R)=\int_{B(0, R) \cap \Omega}\left|\nabla v_{m}\right|^{p} d x$, then $F(R)$ is a continuous function of $R$ satisfying

$$
\lim _{R \rightarrow+\infty} F(R)=\int_{\Omega}\left|\nabla v_{m}\right|^{p} d x, \quad \lim _{R \rightarrow 0} F(R)=0
$$

Up to a subsequence, we can choose minimal $\frac{1}{k_{m}}>0$ such that

$$
\int_{B\left(0, \frac{1}{k_{m}}\right) \cap \Omega}\left|\nabla v_{m}\right|^{p} d x=\delta
$$

We denote by $E: W^{1, p}(\Omega) \rightarrow W^{1, p}\left(\mathbb{R}^{n}\right)$ the extension operator such that

$$
E(v)_{\mid \Omega}=v, \quad\|E(v)\|_{W^{1, p}\left(\mathbb{R}^{n}\right)} \leq C(\Omega)\|v\|_{W^{1, p}(\Omega)}
$$


(remember that $\partial \Omega \in C^{1}$ ). For the simplicity of notations, we shall denote by the same symbol both the function $v \in W^{1, p}(\Omega)$ and its extension $E(v) \in W^{1, p}\left(\mathbb{R}^{n}\right)$. Define

$$
\bar{v}_{m}:=k_{m}^{\frac{p-n}{p}} v_{m}\left(\frac{x}{k_{m}}\right) \text { and } \Omega_{1, m}:=\left\{x \in \mathbb{R}^{n} \mid \frac{x}{k_{m}} \in \Omega\right\}
$$

then $\int_{B(0,1) \cap \Omega_{1, m}}\left|\nabla \bar{v}_{m}\right|^{p} d x=\delta$. Let us point out that, thanks to (2.7)-(2.9), the sequence $\left\{k_{m}\right\}$ is bounded away from zero.

Obviously $\bar{v}_{m} \in W^{1, p}\left(\Omega_{1, m}\right) \subset D^{1, p}\left(\mathbb{R}^{n}\right)$. Moreover,

$$
\left\|\bar{v}_{m}\right\|_{D^{1, p}\left(\mathbb{R}^{n}\right)}=\left\|v_{m}\right\|_{D^{1, p}\left(\mathbb{R}^{n}\right)} \leq C(\Omega)\left\|v_{m}\right\|_{W^{1, p}(\Omega)} \leq c .
$$

Up to a subsequence, there exists $v_{0} \in D^{1, p}\left(\mathbb{R}^{n}\right)$ such that $\bar{v}_{m} \rightarrow v_{0}$ weakly in $D^{1, p}\left(\mathbb{R}^{n}\right)$ and $\bar{v}_{m} \rightarrow v_{0}$ a.e. in $\mathbb{R}^{n}$ as $m \rightarrow+\infty$. We have either $v_{0} \neq \equiv 0$ or $v_{0} \equiv 0$.

Case (I): Assume $v_{0} \neq \equiv 0$.

Since $v_{m} \rightarrow 0(m \rightarrow+\infty)$ weakly in $W^{1, p}(\Omega)$ and $\bar{v}_{m} \rightarrow v_{0} \not \equiv 0$ weakly in $W^{1, p}(\Omega)$, we have $k_{m} \rightarrow+\infty(m \rightarrow+\infty)$.

In this case we claim that $v_{0}$ satisfies (1.6) and the sequence

$$
w_{m}(x):=v_{m}(x)-k_{m}^{\frac{n-p}{p}} v_{0}\left(k_{m} x\right), \quad x \in \Omega
$$

is a Palais-Smale sequence for $F_{\mu}(u)$ at level $d-\frac{p-s}{(n-s) p} S_{\mu, s}^{\frac{n-s}{p-s}}$.

Since $\bar{v}_{m}$ is bounded in $D^{1, p}\left(\mathbb{R}^{n}\right)$, then

$$
\begin{aligned}
& \bar{v}_{m} \rightarrow v_{0} \text { weakly in } D^{1, p}\left(\mathbb{R}^{n}\right), W_{\text {loc }}^{1, p}\left(\mathbb{R}^{n}\right) \text { as } m \rightarrow+\infty ; \\
& \bar{v}_{m} \rightarrow v_{0} \text { a.e. in } \mathbb{R}^{n} \text { as } m \rightarrow+\infty ; \\
& \bar{v}_{m} \rightarrow v_{0} \text { in } L_{\mathrm{loc}}^{p^{*}(s)-1}\left(\mathbb{R}^{n},|x|^{-s}\right) \text { as } m \rightarrow+\infty ; \\
& \bar{v}_{m} \rightarrow v_{0} \text { in } L_{\mathrm{loc}}^{p-1}\left(\mathbb{R}^{n},|x|^{-p}\right) \text { as } m \rightarrow+\infty ; \\
& \bar{v}_{m} \rightarrow v_{0} \text { in } L_{\mathrm{loc}}^{q}\left(\mathbb{R}^{n}\right), 1<q<p^{*} \text {, as } m \rightarrow+\infty
\end{aligned}
$$

If $0 \in \Omega$, fix a ball $B(x, r)$ and a test function $\phi \in C_{0}^{\infty}(B(x, r))$. Notice that for sufficiently large $m, B(x, r) \subset \Omega_{1, m}$. Then we have

$$
\begin{aligned}
& \int_{\Omega_{1, m}}\left|\nabla \bar{v}_{m}\right|^{p-2} \nabla \bar{v}_{m} \nabla \phi d x=\int_{B(x, r)}\left|\nabla \bar{v}_{m}\right|^{p-2} \nabla \bar{v}_{m} \nabla \phi d x \\
& \rightarrow \int_{B(x, r)}\left|\nabla v_{0}\right|^{p-2} \nabla v_{0} \nabla \phi d x ; \\
& \int_{\Omega_{1, m}} \frac{\left|v_{m}\right|^{p^{*}(s)-2} v_{m} \bar{\phi}_{m}}{|x|^{s}} d x=\int_{B(x, r)} \frac{\left|v_{m}\right|^{p^{*}(s)-2} v_{m} \bar{\phi}_{m}}{|x|^{s}} d x \rightarrow \int_{B(x, r)} \frac{\left|v_{0}\right|^{p^{*}(s)-2} v_{0} \phi}{|x|^{s}} d x ; \\
& \int_{\Omega_{1, m}} \mu \frac{\left|\bar{v}_{m}\right|^{p-2} \bar{v}_{m} \phi}{|x|^{p}} d x=\int_{B(x, r)} \mu \frac{\left|\bar{v}_{m}\right|^{p-2} \bar{v}_{m} \phi}{|x|^{p}} d x \rightarrow \int_{B(x, r)} \mu \frac{\left|v_{0}\right|^{p-2} v_{0} \phi}{|x|^{p}} d x
\end{aligned}
$$

as $m \rightarrow+\infty$. 
And since $k_{m} \rightarrow+\infty$ as $m \rightarrow+\infty$, then

$$
\begin{aligned}
& \frac{\lambda}{k_{m}^{p}} \int_{\Omega_{1, m}} \phi \bar{v}_{m}\left|\bar{v}_{m}\right|^{p-2} d x=\frac{\lambda}{k_{m}^{p}} \int_{B(x, r)} \phi \bar{v}_{m}\left|\bar{v}_{m}\right|^{p-2} d x \rightarrow 0 ; \\
& \frac{\eta}{k_{m}^{n-\frac{n-p}{p} q}} \int_{\Omega_{1, m}} \phi\left|\bar{v}_{m}\right|^{q-2} \bar{v}_{m} d x=\frac{\eta}{k_{m}^{n-\frac{n-p}{p} q}} \int_{B(x, r)} \phi\left|\bar{v}_{m}\right|^{q-2} \bar{v}_{m} d x \rightarrow 0 ; \\
& \frac{1}{k_{m}^{p-1}} \int_{\partial \Omega_{1, m}} \alpha\left(\frac{x}{k_{m}}\right) \phi \bar{v}_{m}\left|\bar{v}_{m}\right|^{p-2} d \sigma=0 .
\end{aligned}
$$

Therefore we have

$$
\begin{aligned}
& \left\langle\phi, D F_{\mu}^{\infty}\left(v_{0}, \mathbb{R}^{n}\right)\right\rangle \\
& =\int_{B(x, r)}\left|\nabla v_{0}\right|^{p-2} \nabla v_{0} \nabla \phi d x-\int_{B(x, r)} \frac{\left|v_{0}\right|^{p^{*}(s)-2} v_{0} \phi}{|x|^{s}} d x-\int_{B(x, r)} \mu \frac{\left|v_{0}\right|^{p-2} v_{0} \phi}{|x|^{p}} d x \\
& =\int_{\Omega_{1, m}}\left|\nabla \bar{v}_{m}\right|^{p-2} \nabla \bar{v}_{m} \nabla \phi d x-\int_{\Omega_{1, m}} \frac{\left|\bar{v}_{m}\right|^{p^{*}(s)-2} \bar{v}_{m} \phi}{|x|^{s}} d x-\int_{\Omega_{1, m}} \mu \frac{\left|\bar{v}_{m}\right|^{p-2} \bar{v}_{m} \phi}{|x|^{p}} d x \\
& +\frac{1}{k_{m}^{p-1}} \int_{\partial \Omega_{1, m}} \alpha\left(\frac{x}{k_{m}}\right) \phi \bar{v}_{m}\left|\bar{v}_{m}\right|^{p-2} d \sigma+\frac{\lambda}{k_{m}^{p}} \int_{\Omega_{1, m}} \phi \bar{v}_{m}\left|\bar{v}_{m}\right|^{p-2} d x \\
& -\frac{\eta}{k_{m}^{n-\frac{n-p}{p} q}} \int_{\Omega_{1, m}} \phi\left|\bar{v}_{m}\right|^{q-2} \bar{v}_{m} d x+o(1) \\
& =\int_{\Omega}\left|\nabla v_{m}\right|^{p-2} \nabla v_{m} \nabla \bar{\phi}_{m} d y-\int_{\Omega} \frac{\left|v_{m}\right|^{p^{*}(s)-2} v_{m} \bar{\phi}_{m}}{|y|^{s}} d y-\mu \int_{\Omega} \frac{\left|v_{m}\right|^{p-2} v_{m} \bar{\phi}_{m}}{|y|^{p}} d y \\
& +\int_{\partial \Omega} \alpha(y) \bar{\phi}_{m} v_{m}\left|v_{m}\right|^{p-2} d \sigma+\lambda \int_{\Omega}\left|v_{m}\right|^{p-2} v_{m} \bar{\phi}_{m} d y \\
& -\eta \int_{\Omega} \bar{\phi}_{m}\left|v_{m}\right|^{q-2} v_{m} d y+o(1) \quad\left(\operatorname{let} y=\frac{x}{k_{m}}\right) \\
& =o(1) \text { as } m \rightarrow+\infty \text {, }
\end{aligned}
$$

where $\bar{\phi}_{m}(x)=k_{m}^{\frac{n-p}{p}} \phi\left(k_{m} x\right)$. Since $\|\phi\|_{D^{1, p}(B(x, r))}=\left\|\bar{\phi}_{m}\right\|_{W^{1, p}(\Omega)}+o(1), v_{0}$ solves (1.6).

If $0 \in \partial \Omega$, fix a ball $B(x, r)$ and a test function $\phi \in C_{0}^{\infty}(B(x, r))$. Notice that for sufficiently large $m, B(x, r) \cap \mathbb{R}_{+}^{n} \subset \Omega_{1, m}$, we have

$$
\begin{aligned}
\langle\phi, & \left.D F_{\mu}^{\infty}\left(v_{0}, \mathbb{R}_{+}^{n}\right)\right\rangle \\
= & \int_{B(x, r) \cap \mathbb{R}_{+}^{n}}\left|\nabla v_{0}\right|^{p-2} \nabla v_{0} \nabla \phi d x-\int_{B(x, r) \cap \mathbb{R}_{+}^{n}} \frac{\left|v_{0}\right|^{p^{*}(s)-2} \nu_{0} \phi}{|x|^{s}} d x \\
& -\int_{B(x, r) \cap \mathbb{R}_{+}^{n}} \mu \frac{\left|v_{0}\right|^{p-2} v_{0} \phi}{|x|^{p}} d x \\
= & \int_{\Omega_{1, m}}\left|\nabla \bar{v}_{m}\right|^{p-2} \nabla \bar{v}_{m} \nabla \phi d x-\int_{\Omega_{1, m}} \frac{\left|\bar{v}_{m}\right|^{p^{*}(s)-2} \bar{v}_{m} \phi}{|x|^{s}} d x-\int_{\Omega_{1, m}} \mu \frac{\left|\bar{v}_{m}\right|^{p-2} \bar{v}_{m} \phi}{|x|^{p}} d x \\
& +\frac{1}{k_{m}^{p-1}} \int_{\partial \Omega_{1, m}} \alpha\left(\frac{x}{k_{m}}\right) \phi \bar{v}_{m}\left|\bar{v}_{m}\right|^{p-2} d \sigma+\frac{\lambda}{k_{m}^{p}} \int_{\Omega_{1, m}} \phi \bar{v}_{m}\left|\bar{v}_{m}\right|^{p-2} \bar{v}_{m} d x
\end{aligned}
$$




$$
\begin{aligned}
& -\frac{\eta}{k_{m}^{n-\frac{n-p}{p} q}} \int_{\Omega_{1, m}} \phi\left|\bar{v}_{m}\right|^{q-2} \bar{v}_{m} d x+o(1) \\
= & \int_{\Omega}\left|\nabla v_{m}\right|^{p-2} \nabla v_{m} \nabla \bar{\phi}_{m} d x-\int_{\Omega} \frac{\left|v_{m}\right|^{p^{*}(s)-2} v_{m} \bar{\phi}_{m}}{|x|^{s}} d x-\int_{\Omega} \mu \frac{\left|v_{m}\right|^{p-2} v_{m} \bar{\phi}_{m}}{|x|^{p}} d x \\
& +\int_{\partial \Omega} \alpha(x) \bar{\phi}_{m} v_{m}\left|v_{m}\right|^{p-2} d \sigma+\lambda \int_{\Omega}\left|v_{m}\right|^{p-2} v_{m} \bar{\phi}_{m} d x-\eta \int_{\Omega} \bar{\phi}_{m}\left|v_{m}\right|^{q-2} v_{m} d x+o(1) \\
= & o(1) \quad \text { as } m \rightarrow+\infty
\end{aligned}
$$

where $\bar{\phi}_{m}(x)=k_{m}^{\frac{n-p}{p}} \phi\left(k_{m} x\right)$. Since $\|\phi\|_{D^{1, p}(B(x, r))}=\left\|\bar{\phi}_{m}\right\|_{W^{1, p}(\Omega)}+o(1), v_{0}$ solves $(1.8)$.

By Lemma A.6 in the Appendix and the invariance of dilation, we have for large $m$

$$
\begin{aligned}
& F_{\mu}\left(w_{m}\right)=F_{\mu}\left(v_{m}\right)-F_{\mu}^{\infty}\left(v_{0}\right)+o(1)=d-\frac{p-s}{(n-s) p} S_{\mu, s}^{\frac{n-s}{p-s}}, \quad \text { for } 0 \in \Omega, \\
& F_{\mu}\left(w_{m}\right)=F_{\mu}\left(v_{m}\right)-F_{+, \mu}^{\infty}\left(v_{0}\right)+o(1)=d-\frac{p-s}{2(n-s) p} S_{\mu, s}^{\frac{n-s}{p-s}}, \quad \text { for } 0 \in \partial \Omega, \\
& D F_{\mu}\left(w_{m}\right) \rightarrow 0 \quad \text { in } W^{-1, p}(\Omega) .
\end{aligned}
$$

Also, from $k_{m}^{\frac{n-p}{p}} v_{0}\left(k_{m} x\right) \rightarrow 0$ weakly in $W^{1, p}(\Omega)$ and $v_{m} \rightarrow 0$ weakly in $W^{1, p}(\Omega)$, it is obvious that $w_{m} \rightarrow 0$ weakly in $W^{1, p}(\Omega)$.

Case (II): Assume $v_{0} \equiv 0$.

If $0 \in \Omega$, let $h \in C_{0}^{\infty}(B(0,1))$, then we have

$$
\begin{aligned}
\int_{\mathbb{R}^{n}}\left|\nabla\left(\bar{v}_{m} h\right)\right|^{p} d x \\
=\int_{\mathbb{R}^{n}}\left|\nabla \bar{v}_{m}\right|^{p} h^{p} d x+o(1) \\
=\left\langle D F_{\mu}\left(\bar{v}_{m}\right), h^{p} \bar{v}_{m}\right\rangle+\int_{\mathbb{R}^{n}} \frac{\mu h^{p} \bar{v}_{m}^{p}}{|x|^{p}} d x+\int_{\mathbb{R}^{n}} \frac{\left|\bar{v}_{m}\right|^{p^{*}(s)} h^{p}}{|x|^{s}} d x+o(1) \\
\leq \frac{p^{p} \mu}{(n-p)^{p}} \int_{\mathbb{R}^{n}}\left|\nabla\left(\bar{v}_{m} h\right)\right|^{p} d x+S_{0, s}^{-1}\left(\int_{B(0,1)} \frac{\left|\bar{v}_{m}\right|^{p^{*}(s)}}{|x|^{s}} d x\right)^{\frac{p-s}{n-s}} \int_{\mathbb{R}^{n}}\left|\nabla\left(\bar{v}_{m} h\right)\right|^{p} d x \\
\quad+o(1) \quad \text { as } m \rightarrow+\infty .
\end{aligned}
$$

Choose $\delta$ suitably small, from (2.13) and the fact that $0 \leq \mu<\frac{(n-p)^{p}}{p^{p}}$, we can find $a \in(0,1)$ such that

$$
\int_{B(0, a)}\left|\nabla \bar{v}_{m}\right|^{p} d x \rightarrow 0 \quad \text { as } m \rightarrow+\infty
$$

Thus we have

$$
\int_{B(0, a)} \frac{\left|\bar{v}_{m}\right|^{p}}{|x|^{p}} d x \rightarrow 0, \quad \int_{B(0, a)} \frac{\left|\bar{v}_{m}\right|^{p^{*}(s)}}{|x|^{s}} d x \rightarrow 0 \quad \text { as } m \rightarrow+\infty .
$$

If $0 \in \partial \Omega$, define

$$
\bar{v}_{m}^{\prime}= \begin{cases}v_{m}\left(x^{\prime}, x_{n}\right), & x_{n} \geq 0 \\ v_{m}\left(x^{\prime},-x_{n}\right), & x_{n}<0\end{cases}
$$


where $x^{\prime}=\left(x_{1}, \ldots, x_{n-1}\right)$. Proceeding as to obtain (2.14), we deduce

$$
\int_{B(0, a)}\left|\nabla \bar{v}_{m}^{\prime}\right|^{p} d x \rightarrow 0 \quad \text { as } m \rightarrow+\infty \text { for some } a \in(0,1)
$$

which implies that

$$
\int_{B(0, a) \cap \mathbb{R}_{+}^{n}}\left|\nabla \bar{v}_{m}\right|^{p} d x \rightarrow 0 \quad \text { as } m \rightarrow+\infty \text { for some } a \in(0,1) .
$$

For $p>s>0$, we can deduce that Case (II) cannot happen.

In fact, if $0 \in \Omega$, from (2.10) and $0<p<p^{*}, 0<p^{*}(s)<p^{*}$, then $\forall R>0$,

$$
\begin{aligned}
& \int_{B(0,2) \backslash B(0, a)} \frac{\left|\bar{v}_{m}\right|^{p}}{|x|^{p}} d x \leq \int_{B(0,2) \backslash B(0, a)} \frac{\left|\bar{v}_{m}\right|^{p}}{a^{p}} d x=o(1) \quad \text { as } m \rightarrow+\infty, \\
& \int_{B(0,2) \backslash B(0, a)} \frac{\left|\bar{v}_{m}\right|^{p^{*}(s)}}{|x|^{s}} d x \leq \int_{B(0,2) \backslash B(0, a)} \frac{\left|\bar{v}_{m}\right|^{p^{*}(s)}}{a^{s}} d x=o(1) \quad \text { as } m \rightarrow+\infty .
\end{aligned}
$$

From (2.14)-(2.17), we have

$$
\int_{B(0,2)} \frac{\left|\bar{v}_{m}\right|^{p}}{|x|^{p}} d x=\int_{B(0,2)} \frac{\left|\bar{v}_{m}\right|^{p^{*}(s)}}{|x|^{s}} d x=o(1) \quad \text { as } m \rightarrow+\infty .
$$

Since $\delta>0$, from (2.9) there exists a positive constant $\bar{a}$ such that $k_{m} \geq \bar{a}>0$, thus $B\left(0, \frac{2}{k_{m}}\right) \subset B\left(0, \frac{2}{\bar{a}}\right)$. Choose

$$
0<g_{m} \in C_{0}^{\infty}(\Omega), \quad \operatorname{supp} g_{m} \subset B\left(0, \frac{2}{k_{m}}\right), \quad \text { and } \quad g_{m} \equiv 1 \quad \text { in } B\left(0, \frac{1}{k_{m}}\right) \text {, }
$$

and $g_{m}$ is bounded in $C_{0}^{\infty}(\Omega)$ since $v_{m}$ is the Palais-Smale sequence of $F_{\mu}(u)$, then

$$
\left\langle F_{\mu}^{\prime}\left(v_{m}\right), v_{m} g_{m}\right\rangle=o(1) \quad \text { as } m \rightarrow+\infty
$$

a.e.

$$
\begin{aligned}
& \int_{\Omega}\left|\nabla v_{m}\right|^{p-2} \nabla v_{m} \nabla\left(v_{m} g_{m}\right) d x \\
& =\mu \int_{\Omega} \frac{\left|v_{m}\right|^{p} g_{m}}{|x|^{p}} d x+\int_{\Omega} \frac{\left|v_{m}\right|^{p^{*}(s)} g_{m}}{|x|^{s}} d x-\int_{\partial \Omega} \alpha(x)\left|v_{m}\right|^{p} g_{m} d x \\
& \quad+\eta \int_{\Omega}\left|v_{m}\right|^{q} g_{m} d x-\lambda \int\left|v_{m}\right|^{p} g_{m} d x .
\end{aligned}
$$

Since

$$
\begin{aligned}
& v_{m} \rightarrow 0 \text { weakly in } W^{1, p}(\Omega) \text { as } m \rightarrow+\infty ; \\
& v_{m} \rightarrow 0 \text { in } L^{q}(\Omega), L^{p}(\partial \Omega), 1<q<p *, \text { as } m \rightarrow+\infty ; \\
& v_{m} \rightarrow 0 \text { a.e. in } \Omega \text { as } m \rightarrow+\infty,
\end{aligned}
$$


then from (2.18)-(2.21) we have

$$
\begin{aligned}
\int_{B\left(0, \frac{1}{k_{m}}\right)}\left|\nabla v_{m}\right|^{p} d x \\
\leq \int_{\Omega}\left|\nabla v_{m}\right|^{p} d x \\
\leq \int_{\Omega}\left|\nabla v_{m}\right|^{p-1}\left|v_{m}\right|\left|\nabla g_{m}\right| d x+|\lambda| \int_{\Omega}\left|v_{m}\right|^{p}\left|g_{m}\right| d x \\
\quad+c \int_{B\left(0, \frac{2}{k_{m}}\right)} \frac{\left|v_{m}\right|^{p}}{|x|^{p}} d x+c \int_{B\left(0, \frac{2}{k_{m}}\right)} \frac{\left|v_{m}\right|^{p *(s)}}{|x|^{s}} d x+c \int_{\partial \Omega} \alpha(x)\left|v_{m}\right|^{p} d x+c \eta \int_{\Omega}\left|v_{m}\right|^{q} d x \\
\leq c\left(\int_{\Omega}\left|\nabla v_{m}\right|^{p} d x\right)^{\frac{p-1}{p}}\left(\int_{\Omega}\left|v_{m}\right|^{p} d x\right)^{\frac{1}{p}}+c \int_{B(0,2)} \frac{\left|\bar{v}_{m}\right|^{p}}{|x|^{p}} d x \\
\quad+c \int_{B(0,2)} \frac{\left|\bar{v}_{m}\right|^{p^{*}(s)}}{|x|^{s}} d x+o(1) \\
=o(1) \text { as } m \rightarrow+\infty,
\end{aligned}
$$

where $c$ is a positive constant. Then we have

$$
\left\|v_{m}\right\|_{D^{1, p}\left(B\left(0, \frac{1}{k_{m}}\right)\right)}=\left\|\bar{v}_{m}\right\|_{D_{1, p}(B(0,1))}=o(1) \quad \text { as } m \rightarrow+\infty
$$

which contradicts (2.9).

If $0 \in \partial \Omega$, similarly as (2.16), (2.17), we have

$$
\begin{aligned}
& \int_{B(0,2) \backslash B(0, a)} \frac{\left|\bar{v}_{m}^{\prime}\right|^{p}}{|x|^{p}} d x=o(1) \quad \text { as } m \rightarrow+\infty, \\
& \int_{B(0,2) \backslash B(0, a)} \frac{\left|\bar{v}_{m}^{\prime}\right|^{p^{*}(s)}}{|x|^{s}} d x=o(1) \quad \text { as } m \rightarrow+\infty,
\end{aligned}
$$

then we obtain (2.18).

Thus

$$
\left\|v_{m}\right\|_{D^{1, p\left(B\left(0, \frac{1}{k_{m}}\right) \cap \Omega\right)}}=o(1) \quad \text { as } m \rightarrow+\infty
$$

which contradicts (2.9).

For the case that $s=0$, we denote $\bar{v}_{m}$ by $z_{m}$.

Denote by

$$
Q_{m}(1)=\sup _{x \in \Omega_{1, m}} \int_{B(x, r)}\left|\nabla z_{m}\right|^{p} d x
$$

the concentration function of $z_{m}$. From (2.7), (2.8) we can choose $x_{m} \in \bar{\Omega}_{m}, r_{m} \in \mathbb{R}$ and define

$$
\bar{z}_{m}(x):=r_{m}^{\frac{p-n}{p}} z_{m}\left(\frac{x}{r_{m}}+x_{m}\right)
$$


so that

$$
\bar{Q}_{m}(1)=\sup _{\frac{x}{r_{m}}+x_{m} \in \Omega_{1, m}} \int_{B(x, 1)}\left|\nabla \bar{z}_{m}\right|^{p} d x=\int_{B(0,1)}\left|\nabla \bar{z}_{m}\right|^{p} d x=\delta_{1} \leq \frac{1}{2 L} S_{\mu}^{\frac{n}{p}},
$$

where $0<\delta_{1}<\delta, L$ denotes the least number of balls with radius 1 in $\mathbb{R}^{n}$ that are needed to cover a ball of radius 2 .

Note that there exists a constant $b>0$ such that $r_{m} \geq b$. Set

$$
\tilde{\Omega}_{m}:=\left\{x \in \mathbb{R}^{n} \mid \frac{x}{r_{m}}+x_{m} \in \Omega_{1, m}\right\} .
$$

We may assume $\bar{z}_{m} \in D^{1, p}\left(\mathbb{R}^{n}\right)$. Moreover, $\left\{\bar{z}_{m}\right\}$ is bounded uniformly in $D^{1, p}\left(\mathbb{R}^{n}\right)$. Thus, up to a subsequence,

$$
\bar{z}_{m} \rightarrow \bar{v}_{0} \text { weakly in } D^{1, p}\left(\mathbb{R}^{n}\right) \text { as } m \rightarrow+\infty .
$$

We are going to prove that the convergence actually holds in the strong $W_{\text {loc }}^{1, p}\left(\mathbb{R}^{n}\right)$ sense.

Since $C_{0}^{\infty}\left(\mathbb{R}^{n}\right) \cap W_{\text {loc }}^{1, p}\left(\mathbb{R}^{n}\right)$ is dense in $W_{\text {loc }}^{1, p}\left(\mathbb{R}^{n}\right)$, then without loss of generality we can assume that $\bar{v}_{m}-\bar{v}_{0} \in C_{0}^{\infty}\left(\mathbb{R}^{n}\right) \cap W_{\text {loc }}^{1, p}\left(\mathbb{R}^{n}\right)$. Let $x_{0}$ be a fixed point of $\mathbb{R}^{n}$, from Proposition 6.6 in [19], we can find $\rho \in[1,2]$ such that the solution $\bar{w}_{m}$ of the Dirichlet problem

$$
\left\{\begin{array}{l}
\Delta_{p} w=0 \quad \text { in } B\left(x_{0}, 3\right) \backslash B\left(x_{0}, \rho\right), \\
w_{\mid \partial B\left(x_{0}, \rho\right)}=\bar{v}_{m}-\bar{v}_{0}, \quad w_{\mid \partial B\left(x_{0}, 3\right)}=0
\end{array}\right.
$$

satisfies the following conditions:

$$
\bar{w}_{m} \rightarrow 0 \text { in } W^{1, p}\left(B\left(x_{0}, 3\right) \backslash B\left(x_{0}, \rho\right)\right) \text { as } m \rightarrow+\infty \text {. }
$$

Define

$$
\varphi_{m}= \begin{cases}\bar{v}_{m}-\bar{v}_{0} & \text { in } B\left(x_{0}, \rho\right), \\ \bar{w}_{m} & \text { in } B\left(x_{0}, 3\right) \backslash B\left(x_{0}, \rho\right), \\ 0 & \text { in } \mathbb{R}^{n} \backslash B\left(x_{0}, 3\right) .\end{cases}
$$

It follows from the above equation that $\left\|\varphi_{m}\right\|_{L^{p}\left(\mathbb{R}^{n}\right)} \rightarrow 0$ as $m \rightarrow+\infty$. Now, scaling back the function $\varphi_{m}$,

$$
\bar{\varphi}_{m}=r_{m}^{\frac{n-p}{p}} \varphi_{m}\left(r_{m}\left(x-x_{m}\right)\right),
$$

then there exists a constant $\beta>0$ such that supp $\bar{\varphi}_{m} \subset B\left(x_{0}, \beta\right) \subset \Omega_{1, m}$ for $m$ large.

Taking into account (2.27), (2.28) and (2.29), letting $m \rightarrow+\infty$, we have

$$
\left\|\nabla \bar{\varphi}_{m}\right\|_{L^{p}\left(B\left(x_{0}, \beta\right)\right)}^{p}-\left\|\varphi_{m}\right\|_{D^{1, p}\left(\mathbb{R}^{n}\right)}^{p}-\left\|\bar{z}_{m}-\bar{v}_{0}\right\|_{D^{1, p}\left(B\left(x_{0}, \rho\right)\right)}^{p} \rightarrow 0 .
$$

By scale invariance and the fact that $\left\{z_{m}\right\}$ is a Palais-Smale sequence for $F_{\mu}(u)$, it follows that

$$
\left\langle D F_{\mu, m}\left(\bar{z}_{m}\right), \varphi_{m}\right\rangle=\left\langle D F_{\mu}\left(z_{m}\right), \bar{\varphi}_{m}\right\rangle+o(1)=o(1),
$$


where

$$
\begin{aligned}
F_{\mu, m}(\bar{v})= & \frac{1}{p} \int_{\tilde{\Omega}_{m}}\left(|\nabla \bar{v}|^{p}-\mu \frac{|\overline{\mid}|^{p}}{\left|x+r_{m} x_{m}\right|^{p}}\right) d x+\frac{1}{p r_{m}^{p-1}} \int_{\partial \tilde{\Omega}_{m}} \alpha\left(x_{m}+\frac{x}{r_{m}}\right)|\bar{v}|^{p} d \sigma \\
& -\frac{1}{p^{*}} \int_{\tilde{\Omega}_{m}}|\bar{v}|^{p^{*}} d x-\frac{\eta}{q r_{m}^{n-\frac{n-p}{p} q}} \int_{\tilde{\Omega}_{m}}|\bar{v}|^{q} d x+\frac{\lambda}{p r_{m}^{p}} \int_{\tilde{\Omega}_{m}}|\bar{v}|^{p} d x .
\end{aligned}
$$

Therefore, from the definitions of $F_{\mu, m}, \varphi_{m}$ and (2.28), we have

$$
\begin{aligned}
o(1)= & \int_{\tilde{\Omega}_{m} \cap B\left(x_{0}, \rho\right)}\left[\left|\nabla \bar{z}_{m}\right|^{p-2} \nabla \bar{z}_{m} \nabla\left(\bar{z}_{m}-\bar{v}_{0}\right)-\mu \frac{\left|\bar{z}_{m}\right|^{p-2} \bar{z}_{m}\left(\bar{z}_{m}-\bar{v}_{0}\right)}{\left|x+r_{m} x_{m}\right|^{p}}\right] d x \\
& -\int_{\tilde{\Omega}_{m} \cap B\left(x_{0}, \rho\right)}\left|\bar{z}_{m}\right|^{p^{*}-2} \bar{z}_{m}\left(\bar{z}_{m}-\bar{v}_{0}\right) d x+o(1) \\
= & \int_{\tilde{\Omega}_{m} \cap B\left(x_{0}, \rho\right)}\left(\left|\nabla\left(\bar{z}_{m}-\bar{v}_{0}\right)\right|^{p}-\mu \frac{\left|\bar{z}_{m}-\bar{v}_{0}\right|^{p}}{\left|x+r_{m} x_{m}\right|^{p}}\right) d x \\
& -\int_{\tilde{\Omega}_{m} \cap B\left(x_{0}, \rho\right)}\left|\bar{z}_{m}-\bar{v}_{0}\right|^{p^{*}} d x+o(1) \\
= & \int_{\tilde{\Omega}_{m}}\left(\left|\nabla \varphi_{m}\right|^{p}-\mu \frac{\left|\varphi_{m}\right|^{p}}{\left|x+r_{m} x_{m}\right|^{p}}\right) d x-\int_{\tilde{\Omega}_{m}}\left|\varphi_{m}\right|^{p^{*}} d x+o(1) .
\end{aligned}
$$

Moreover, by scale invariance and

$$
\begin{aligned}
& \int_{B\left(x_{0}, \beta\right)}\left|\bar{\varphi}_{m}\right|^{p} d x=\int_{\Omega_{1, m}}\left|\bar{\varphi}_{m}\right|^{p} d x=\frac{1}{r_{m}^{p}} \int_{\mathbb{R}^{n}}\left|\varphi_{m}\right|^{p} d x=o(1) \text { as } m \rightarrow+\infty, \\
& o(1)=\int_{\Omega_{1, m}}\left(\left|\nabla \bar{\varphi}_{m}\right|^{p}-\mu \frac{\left|\bar{\varphi}_{m}\right|^{p}}{|x|^{p}}\right) d x-\int_{\Omega_{1, m}}\left|\bar{\varphi}_{m}\right|^{p^{*}} d x \\
& \geq \int_{\Omega_{1, m}}\left(\left|\nabla \bar{\varphi}_{m}\right|^{p}-\mu \frac{\left|\bar{\varphi}_{m}\right|^{p}}{|x|^{p}}\right) d x\left(1-\frac{\left\|\bar{\varphi}_{m}\right\|_{L^{p^{*}}\left(\Omega_{1, m}\right)}^{p^{*}}}{\int_{\Omega_{1, m}}\left(\left.\nabla \bar{\varphi}_{m}\right|^{p}-\mu \frac{\left|\bar{\varphi}_{m}\right|^{p}}{|x|^{p}}\right) d x}\right) \\
& \geq \int_{\Omega_{1, m}}\left(\left|\nabla \bar{\varphi}_{m}\right|^{p}-\mu \frac{\left|\bar{\varphi}_{m}\right|^{p}}{|x|^{p}}\right) d x\left(1-\frac{\left\|\nabla \bar{\varphi}_{m}\right\|_{L^{p}\left(\Omega_{1, m}\right)}^{p^{*}-p}}{S_{\mu}^{\frac{p^{*}}{p}}}\right) \\
& \geq \int_{\Omega_{1, m}}\left(\left|\nabla \bar{\varphi}_{m}\right|^{p}-\mu \frac{\left|\bar{\varphi}_{m}\right|^{p}}{|x|^{p}}\right) d x\left(1-\frac{\left\|\nabla\left(\bar{z}_{m}-\bar{v}_{0}\right)\right\|_{L^{p}\left(B\left(x_{0}, \rho\right)\right)}^{p^{*}-p}}{S_{\mu}^{\frac{p^{*}}{p}}}\right) .
\end{aligned}
$$

Let us cover $B\left(x_{0}, \rho\right)$ with $L$ balls of radius one, from (2.26) then

$$
\begin{aligned}
\left\|\nabla\left(\bar{z}_{m}-\bar{v}_{0}\right)\right\|_{L^{p}\left(B\left(x_{0}, \rho\right)\right)}^{p} & \leq\left\|\nabla \bar{z}_{m}\right\|_{L^{p}\left(B\left(x_{0}, \rho\right)\right)}^{p}+o(1) \\
& \leq L\left\|\nabla \bar{z}_{m}\right\|_{L^{p}(B(0,1))}^{p}+o(1) \leq \frac{1}{2} S_{\mu}^{n / p}+o(1),
\end{aligned}
$$

so that (2.31) and (2.32) yield

$$
\left\|\bar{\varphi}_{m}\right\|_{W^{1, p}\left(B\left(x_{0}, \beta\right)\right)}=\left\|\bar{\varphi}_{m}\right\|_{W^{1, p}\left(\Omega_{1, m}\right)} \rightarrow 0 \quad \text { as } m \rightarrow+\infty .
$$


Finally, using again the properties of the extension operator, we obtain from (2.30)

$$
\begin{aligned}
\left\|\bar{\varphi}_{m}\right\|_{W^{1, p}\left(B\left(x_{0}, \beta\right)\right)}^{p} & \geq \frac{1}{C^{p}}\left\|\bar{\varphi}_{m}\right\|_{W^{1, p}\left(\mathbb{R}^{n}\right)}^{p} \\
& =\frac{1}{C^{p}}\left\|\varphi_{m}\right\|_{W^{1, p}\left(\mathbb{R}^{n}\right)}^{p}+o(1) \\
& =\frac{1}{C^{p}}\left\|\bar{z}_{m}-\bar{v}_{0}\right\|_{W^{1, p}\left(B\left(x_{0}, \rho\right)\right)}^{p}+o(1) \text { as } m \rightarrow+\infty,
\end{aligned}
$$

where $C$ is a positive constant depending on the domain $B\left(x_{0}, \beta\right)$. Therefore

$$
\forall x_{0} \in \mathbb{R}^{n}, \quad\left\|\bar{z}_{m}-\bar{v}_{0}\right\|_{W^{1, p}\left(B\left(x_{0}, \rho\right)\right)} \rightarrow 0 .
$$

Since $\int_{B(0,1)}\left|\nabla \bar{z}_{m}\right|^{p} d x=\delta_{1}>0$, we have $\bar{v}_{0} \not \equiv 0$. Hence by local properties of the extension operator, we have that $\left.\bar{v}_{0}\right|_{\tilde{\Omega}_{m}} \nrightarrow 0$. Since $z_{m} \rightarrow 0$ weakly in $D^{1, p}\left(\mathbb{R}^{n}\right)$, we also have $r_{m} \rightarrow+\infty$ as $m \rightarrow+\infty$.

Now, using the result of Case (I), we have

$$
\bar{z}_{m}(x)=r_{m}^{\frac{p-n}{p}} \bar{v}_{m}\left(\frac{x}{r_{m}}+x_{m}\right)=\left(r_{m} k_{m}\right)^{\frac{p-n}{p}} v_{m}\left(\frac{x}{r_{m} k_{m}}+\frac{x_{m}}{k_{m}}\right) .
$$

Define $K_{m}=r_{m} k_{m}, y_{m}=x_{m} / k_{m}$; then $y_{m} \rightarrow y_{0} \in \bar{\Omega}, K_{m}\left|y_{m}\right|=r_{m}\left|x_{m}\right|$. By (2.14) we have $\left|x_{m}\right|>a>0$, so $K_{m}\left|y_{m}\right| \rightarrow+\infty$. Also, by the fact that $\left\{k_{m}\right\}$ is bounded away from zero, $K_{m} \rightarrow+\infty($ as $m \rightarrow+\infty)$. Then $\tilde{\Omega}_{m}=\left\{x \in \mathbb{R}^{n} \mid \frac{x}{K_{m}}+y_{m} \in \Omega\right\}, \bar{v}_{m}=K_{m}^{\frac{p-n}{p}} v_{m}\left(\frac{x}{K_{m}}+y_{m}\right)$.

Since we have $\int_{\tilde{\Omega}_{m}} \frac{\left|\bar{z}_{m}\right|^{p-2} \bar{z}_{m} \phi}{\left|x+K_{m} y_{m}\right|^{p}} d x=o(1)$ for large $m$ and any given $\phi \in C_{0}^{\infty}(B(x, r))$, we can proceed our proof as follows.

(1) For the case when $\lim _{m \rightarrow+\infty} K_{m} \operatorname{dist}\left(y_{m}, \partial \Omega\right)=+\infty$ uniformly, we claim that $\bar{v}_{0}$ solves (1.5). Indeed, for a fixed ball $B(x, r)$ and a test function $\phi \in C_{0}^{\infty}(B(x, r))$ and for sufficiently large $m, B(x, r) \subset \tilde{\Omega}_{m}$. Therefore, we have

$$
\begin{aligned}
\langle\phi, & \left.D F^{\infty}\left(\bar{v}_{0}, \mathbb{R}^{n}\right)\right\rangle \\
= & \int_{B(x, r)}\left|\nabla \bar{v}_{0}\right|^{p-2} \nabla \bar{v}_{0} \nabla \phi d x-\int_{B(x, r)}\left|\bar{v}_{0}\right|^{p^{*}-2} \bar{v}_{0} \phi d x \\
= & \int_{\tilde{\Omega}_{m}}\left|\nabla \bar{z}_{m}\right|^{p-2} \nabla \bar{z}_{m} \nabla \phi d x-\int_{\tilde{\Omega}_{m}}\left|\bar{z}_{m}\right|^{p^{*}-2} \bar{z}_{m} \phi d x-\int_{\tilde{\Omega}_{m}} \mu \frac{\left|\bar{z}_{m}\right|^{p-2} \bar{z}_{m} \phi}{\left|x+K_{m} y_{m}\right|^{p}} d x \\
& -\frac{\eta}{K_{m}^{n-\frac{n-p}{p} q}} \int_{\tilde{\Omega}_{m}} \phi\left|\bar{z}_{m}\right|^{q-2} \bar{z}_{m} d x+\frac{1}{K_{m}^{p-1}} \int_{\partial \tilde{\Omega}_{m}} \alpha\left(\frac{x}{K_{m}}+y_{m}\right) \phi \bar{z}_{m}\left|\bar{z}_{m}\right|^{p-2} d \sigma \\
& +\frac{\lambda}{K_{m}^{p}} \int_{\tilde{\Omega}_{m}}\left|\bar{z}_{m}\right|^{p-2} \bar{z}_{m} \phi d x+o(1) \\
= & \int_{\Omega}\left|\nabla v_{m}\right|^{p-2} \nabla v_{m} \nabla \bar{\phi}_{m} d x-\int_{\Omega}\left|v_{m}\right|^{p^{*}-2} v_{m} \bar{\phi}_{m} d x-\int_{\Omega} \mu \frac{\left|v_{m}\right|^{p-2} v_{m} \bar{\phi}_{m}}{|x|^{p}} d x \\
& +\int_{\partial \Omega} \alpha(x) \bar{\phi}_{m} v_{m}\left|v_{m}\right|^{p-2} d \sigma-\eta \int_{\Omega} \bar{\phi}_{m}\left|v_{m}\right|^{q-2} v_{m} d x+\lambda \int_{\Omega}\left|v_{m}\right|^{p-2} v_{m} \bar{\phi}_{m} d x+o(1) \\
= & o(1) \text { as } m \rightarrow+\infty,
\end{aligned}
$$

where $\bar{\phi}_{m}(x)=K_{m}^{\frac{n-p}{p}} \phi\left(K_{m}\left(x-y_{m}\right)\right)$. 
(2) For the case when $\lim _{m \rightarrow+\infty} K_{m} \operatorname{dist}\left(y_{m}, \partial \Omega\right) \rightarrow c<+\infty$, we claim that $\bar{v}_{0}$ solves (1.7). Indeed, fix a ball $B(x, r)$ and a test function $\phi \in C_{0}^{\infty}(B(x, r))$ and note that, for sufficiently large $m, B(x, r) \cap \mathbb{R}_{+}^{n} \subset \tilde{\Omega}_{m}$ we have

$$
\begin{aligned}
\left\langle\phi, D F_{0}^{\infty}\left(\bar{v}_{0}, \mathbb{R}_{+}^{n}\right)\right\rangle & \\
= & \int_{B(x, r) \cap \mathbb{R}_{+}^{n}}\left|\nabla \bar{v}_{0}\right|^{p-2} \nabla \bar{v}_{0} \nabla \phi d x-\int_{B(x, r) \cap \mathbb{R}_{+}^{n}}\left|\bar{v}_{0}\right|^{p^{*}-2} \bar{v}_{0} \phi d x \\
= & \int_{\tilde{\Omega}_{m}}\left|\nabla \bar{z}_{m}\right|^{p-2} \nabla \bar{z}_{m} \nabla \phi d x-\int_{\tilde{\Omega}_{m}}\left|\bar{z}_{m}\right|^{p^{*}-2} \bar{z}_{m} \phi d x-\int_{\tilde{\Omega}_{m}} \mu \frac{\left|\bar{z}_{m}\right|^{p-2} \bar{z}_{m} \phi}{\left|x+K_{m} y_{m}\right|^{p}} d x \\
& -\frac{\eta}{K_{m}^{n-\frac{n-p}{p} q}} \int_{\tilde{\Omega}_{m}} \phi\left|\bar{z}_{m}\right|^{q-2} \bar{z}_{m} d x+\frac{1}{K_{m}^{p-1}} \int_{\partial \tilde{\Omega}_{m}} \alpha\left(\frac{x}{K_{m}}+y_{m}\right) \phi \bar{z}_{m}\left|\bar{z}_{m}\right|^{p-2} d \sigma \\
& +\frac{\lambda}{K_{m}^{p}} \int_{\tilde{\Omega}_{m}}\left|\bar{z}_{m}\right|^{p-2} \bar{z}_{m} \phi d x+o(1) \\
= & \int_{\Omega}\left|\nabla v_{m}\right|^{p-2} \nabla v_{m} \nabla \bar{\phi}_{m} d x-\int_{\Omega}\left|v_{m}\right|^{p^{*}-2} v_{m} \bar{\phi}_{m} d x-\int_{\Omega} \mu \frac{\left|v_{m}\right|^{p-2} v_{m} \bar{\phi}_{m}}{|x|^{p}} d x \\
& +\int_{\partial \Omega} \alpha(x) \bar{\phi}_{m} v_{m}\left|v_{m}\right|^{p-2} d \sigma-\eta \int_{\Omega} \bar{\phi}_{m}\left|v_{m}\right|^{p-2} v_{m} d x+\lambda \int_{\Omega} v_{m} \bar{\phi}_{m} d x+o(1) \\
= & o(1) \text { as } m \rightarrow+\infty,
\end{aligned}
$$

where $\bar{\phi}_{m}(x)=K_{m}^{\frac{n-p}{p}} \phi\left(K_{m}\left(x-y_{m}\right)\right)$.

Define

$$
w_{m}(x)=v_{m}(x)-K_{m}^{\frac{n-p}{p}} \bar{v}_{0}\left(K_{m}\left(x-y_{m}\right)\right) .
$$

For the case that $\lim _{m \rightarrow+\infty} K_{m} \operatorname{dist}\left(y_{m}, \partial \Omega\right)=c<+\infty$, we have that $\bar{v}_{0}$ is a weak solution of equation (1.5) and $w_{m}$ is a Palais-Smale sequence of $F_{\mu}(u)$ at level $d-\frac{1}{2 n} S^{\frac{n}{p}}$. For the case that $\lim _{m \rightarrow+\infty} K_{m} \operatorname{dist}\left(y_{m}, \partial \Omega\right)=+\infty$, we have that $\bar{v}_{0}$ is a weak solution of equation (1.7) and $w_{m}$ is a Palais-Smale sequence of $F_{\mu}(u)$ at level $d-\frac{1}{n} S^{\frac{n}{p}}$.

This concludes the proof of Lemma 2.1.

Now, we are going to complete the proof of Theorem 1.1.

Proof of Theorem 1.1 By applying Lemma 2.1, Lemmas A.4-A.6 recursively, the iteration must stop after a finite number of steps; moreover, the last Palais-Smale sequence must strongly converge to zero. Hence we prove parts (i) and (ii).

As a consequence, we finish the proof of Theorem 1.1.

\section{The proofs of existence results}

In this section, we shall apply Theorem 1.1 and the mountain pass theorem [18] to obtain the existence of critical points for $F_{\mu}(u)$ under different assumptions on the parameters $\mu$, $\lambda$ and the fact that $0 \in \Omega$ or $0 \in \partial \Omega$. For convenience, we only consider the case of $\alpha(x)=0$.

Lemma 3.1 For $\lambda>-\lambda_{1}, F_{\mu}(u)$ satisfies the geometry structure of the mountain pass theorem. 
By Lemma A.3 in the Appendix, the proof of Lemma 3.1 can be completed easily.

Define

$$
c_{\mu}=: \inf _{\gamma \in \Gamma} \sup _{t \in[0,1]} F_{\mu}(\gamma(t))
$$

where $\Gamma=\left\{\gamma \in C\left([0,1], W^{1, p}(\Omega)\right): \gamma(0)=0, \gamma(1)=\psi_{0} \in W^{1, p}(\Omega)\right\}$. The $\psi_{0}$ is chosen such that $F_{\mu}\left(t \psi_{0}\right) \leq 0$ for all $t \geq 1$.

According to Theorem 1.1, we easily have the following.

Proposition 3.1 For the case that $s \neq 0$, the following two statements are true:

(1) Suppose $0 \in \Omega, \mu \in(0, \bar{\mu})$ and $\lambda>-\lambda_{1}$. If

$$
0<c_{\mu}<\frac{p-s}{(n-s) p} S_{\mu, s}^{\frac{n-s}{p-s}}
$$

then (1.1) has a positive solution satisfying $F_{\mu}(u) \leq c_{\mu}$.

(2) Suppose $0 \in \partial \Omega, \mu \in(0, \bar{\mu})$ and $\lambda>-\lambda_{1}$. If

$$
0<c_{\mu}<\frac{p-s}{2(n-s) p} S_{\mu, s}^{\frac{n-s}{p-s}}
$$

then (1.1) has a positive solution satisfying $F_{\mu}(u) \leq c_{\mu}$.

Proposition 3.2 For the case that $s=0$, the following two statements are true:

(1) Suppose $0 \in \Omega, \mu \in(0, \bar{\mu})$ and $\lambda>-\lambda_{1}$. If

$$
0<c_{\mu}<\min \left\{\frac{1}{2 n} S^{\frac{n}{p}}, \frac{1}{n} S_{\mu}^{\frac{n}{p}}\right\},
$$

then (1.1) has a positive solution satisfying $F_{\mu}(u) \leq c_{\mu}$.

(2) Suppose $0 \in \partial \Omega, \mu \in(0, \bar{\mu})$ and $\lambda>-\lambda_{1}$. If

$$
0<c_{\mu}<\frac{1}{2 n} S_{\mu}^{\frac{n}{p}},
$$

then (1.1) has a positive solution satisfying $F_{\mu}(u) \leq c_{\mu}$.

Proof of Theorem 1.2 By Proposition 3.1, we only need to prove that $c_{\mu}<\frac{p-s}{(n-s) p} S_{\mu, s}^{(n-s) /(p-s)}$. Let $\varphi(x) \in C_{0}^{\infty}(\Omega), \varphi(x)=1$ for $|x| \leq R, \varphi(x)=0$ for $|x| \geq 2 R$, where $B(0,2 R) \subset \Omega$. Set $v_{\varepsilon}(x)=$ $\varphi(x) V_{\mu}^{\varepsilon}(x)$, we only need to verify

$$
\max _{t>0} F_{\mu}\left(t v_{\varepsilon}\right)<\frac{p-s}{(n-s) p} S_{\mu, s}^{(n-s) /(p-s)} .
$$

It is easy to get the following estimates (Lemma 2.3 in [17]):

$$
\begin{aligned}
& \int_{\Omega}\left(\left|\nabla v_{\varepsilon}\right|^{p}-\mu \frac{\left|v_{\varepsilon}\right|^{p}}{|x|^{p}}\right) d x=S_{\mu, s}^{(n-s) /(p-s)}+O\left(\varepsilon^{b(\mu) p+p-n}\right) ; \\
& \int_{\Omega} \frac{\left|v_{\varepsilon}\right|^{p^{*}(s)}}{|x|^{s}} d x=S_{\mu, s}^{(n-s) /(p-s)}+O\left(\varepsilon^{b(\mu) p^{*}(s)-n+s}\right) ;
\end{aligned}
$$




$$
\begin{aligned}
\int_{\Omega}\left|v_{\varepsilon}\right|^{p} d x & = \begin{cases}O\left(\varepsilon^{b(\mu) p+p-n}\right), & p<\frac{n}{b(\mu)}, \\
O\left(\varepsilon^{p}|\log \varepsilon|\right), & p=\frac{n}{b(\mu)}, \\
O\left(\varepsilon^{p}\right), & p>\frac{n}{b(\mu)}\end{cases} \\
\int_{\Omega}\left|v_{\varepsilon}\right|^{q} d x & = \begin{cases}O\left(\varepsilon^{\left(b(\mu)+1-\frac{n}{p}\right) q}\right), & q<\frac{n}{b(\mu)}, \\
O\left(\varepsilon^{n+\left(1-\frac{n}{p}\right) q}|\log \varepsilon|\right), & q=\frac{n}{b(\mu)}, \\
O\left(\varepsilon^{n+\left(1-\frac{n}{p}\right) q}\right), & q>\frac{n}{b(\mu)} .\end{cases}
\end{aligned}
$$

Since $\max \left\{p, \frac{n}{b(\mu)}, \frac{p(2 n-b(\mu) p-p)}{n-p}\right\}<q<p^{*}(s)$ and from (3.9), we have

$$
\int_{\Omega}\left|v_{\varepsilon}\right|^{q} d x=O\left(\varepsilon^{n+\left(1-\frac{n}{p}\right) q}\right), O\left(\varepsilon^{p}\right)+O\left(\varepsilon^{p}|\log \varepsilon|\right)+O\left(\varepsilon^{b(\mu) p+p-n}\right)=o\left(\varepsilon^{n+\left(1-\frac{n}{p}\right) q}\right) .
$$

Similar as the proof of Lemma 8.1 in [20], let $t_{\varepsilon}$ be the attaining point of $\max _{t>0} F_{\mu}\left(t v_{\varepsilon}\right)$, we claim $t_{\varepsilon}$ is uniformly bounded for $\varepsilon>0$ small. In fact, we consider the function

$$
\begin{aligned}
g(t)= & F_{\mu}\left(t v_{\varepsilon}\right)=\frac{t^{p}}{p} \int_{\Omega}\left(\left|\nabla v_{\varepsilon}\right|^{p}-\mu \frac{\left|v_{\varepsilon}\right|^{p}}{|x|^{p}}\right) d x-\frac{t^{p^{*}(s)}}{p^{*}(s)} \int_{\Omega} \frac{\left|v_{\varepsilon}\right|^{p^{*}(s)}}{|x|^{s}} d x \\
& +\frac{t^{p}}{p} \int_{\Omega} \lambda\left|v_{\varepsilon}\right|^{p} d x-\eta \frac{t^{q}}{q} \int_{\Omega}\left|v_{\varepsilon}\right|^{q} d x .
\end{aligned}
$$

Since $\lim _{t \rightarrow+\infty} g(t)=-\infty$ and $g(t)>0$ when $t$ is close to 0 , so that $\max _{t>0} g(t)$ is attained for $t_{\varepsilon}>0$. Then

$$
\begin{aligned}
g^{\prime}\left(t_{\varepsilon}\right)= & t_{\varepsilon}^{p-1} \int_{\Omega}\left(\left|\nabla v_{\varepsilon}\right|^{p}-\mu \frac{\left|v_{\varepsilon}\right|^{p}}{|x|^{p}}+\lambda\left|v_{\varepsilon}\right|^{p}\right) d x \\
& -t_{\varepsilon}^{p^{*}(s)-1} \int_{\Omega} \frac{\left|v_{\varepsilon}\right|^{p^{*}(s)}}{|x|^{s}} d x-\eta t_{\varepsilon}^{q-1} \int_{\Omega}\left|v_{\varepsilon}\right|^{q} d x=0 .
\end{aligned}
$$

Since $\eta>0$, from (3.6)-(3.9) and (3.11), for $\varepsilon$ sufficiently small, we have

$$
t_{\varepsilon}^{p^{*}(s)-p}<\frac{\int_{\Omega}\left(\left|\nabla v_{\varepsilon}\right|^{p}-\mu \frac{\left|v_{\varepsilon}\right|^{p}}{|x|^{p}}+\lambda\left|v_{\varepsilon}\right|^{p}\right) d x}{\int_{\Omega} \frac{\mid \nu_{\varepsilon} p^{*}(s)}{|x|^{s}} d x}<2 .
$$

Thus from (3.8), (3.11), (3.12), $p<q<p^{*}(s)$ and for $\varepsilon$ sufficiently small,

$$
\begin{aligned}
& \int_{\Omega}\left(\left|\nabla v_{\varepsilon}\right|^{p}-\mu \frac{\left|v_{\varepsilon}\right|^{p}}{|x|^{p}}+\lambda\left|v_{\varepsilon}\right|^{p}\right) d x \\
& \quad \leq t_{\varepsilon}^{p^{*}(s)-p} \int_{\Omega} \frac{\left|v_{\varepsilon}\right|^{*}(s)}{|x|^{s}} d x+2 \frac{q-p}{p^{*}(s)-p} \eta \int_{\Omega}\left|v_{\varepsilon}\right|^{q} d x \\
& \quad \leq t_{\varepsilon}^{p^{*}(s)-p} \int_{\Omega} \frac{\left|v_{\varepsilon}\right|^{*}(s)}{|x|^{s}} d x+\frac{1}{2} \int_{\Omega}\left(\left|\nabla v_{\varepsilon}\right|^{p}-\mu \frac{\left|v_{\varepsilon}\right|^{p}}{|x|^{p}}+\lambda\left|v_{\varepsilon}\right|^{p}\right) d x .
\end{aligned}
$$

By (3.6)-(3.9), (3.13) and choosing $\varepsilon$ small enough, we have

$$
t_{\varepsilon}^{p^{*}(s)-p} \geq \frac{\frac{1}{2} \int_{\Omega}\left(\left|\nabla v_{\varepsilon}\right|^{p}-\mu \frac{\left|\nu_{\varepsilon}\right|^{p}}{|x|^{p}}+\lambda\left|v_{\varepsilon}\right|^{p}\right) d x}{\int_{\Omega} \frac{\left|\nu_{\varepsilon}\right|^{*}(s)}{|x|^{\mid}} d x}>\frac{1}{4} .
$$

Thus $t_{\varepsilon}$ is uniformly bounded for $\varepsilon>0$ small enough. 
Then from (3.6)-(3.10), (3.12) and (3.14), for $\varepsilon$ sufficiently small, we have

$$
\begin{aligned}
\max _{t>0} F_{\mu}\left(t v_{\varepsilon}\right)= & F_{\mu}\left(t_{\varepsilon} v_{\varepsilon}\right) \\
\leq & \max _{t>0}\left\{\frac{t^{p}}{p} \int_{\Omega}\left(\left|\nabla v_{\varepsilon}\right|^{p}-\mu \frac{\left|v_{\varepsilon}\right|^{p}}{|x|^{p}}\right) d x-\frac{t^{p^{*}(s)}}{p^{*}(s)} \int_{\Omega} \frac{\left|v_{\varepsilon}\right|^{*}(s)}{|x|^{s}} d x\right\} \\
& +\frac{t_{\varepsilon}^{p}}{p} \int_{\Omega} \lambda\left|v_{\varepsilon}\right|^{p} d x-\eta \frac{t_{\varepsilon}^{q}}{q} \int_{\Omega}\left|v_{\varepsilon}\right|^{q} d x \\
= & \frac{p-s}{(n-s) p} S_{\mu, s}^{\frac{n-s}{p-s}}+O\left(\varepsilon^{b(\mu) p+p-n}\right)-O\left(\varepsilon^{b(\mu) p^{*}(s)-n+s}\right) \\
& -\eta \begin{cases}O\left(\varepsilon^{\left(b(\mu)+1-\frac{N}{p}\right) q}\right), & q<\frac{n}{b(\mu)}, \\
O\left(\varepsilon^{n+\left(1-\frac{n}{p}\right) q}|\log \varepsilon|\right), & q=\frac{n}{b(\mu)},+\lambda \begin{cases}O\left(\varepsilon^{b(\mu) p+p-n}\right), & p<\frac{n}{b(\mu)} \\
O\left(\varepsilon^{p}|\log \varepsilon|\right), & p=\frac{n}{b(\mu)} \\
O\left(\varepsilon^{n+\left(1-\frac{n}{p}\right) q}\right), & p>\frac{n}{b(\mu)}\end{cases} \\
< & \frac{p-s}{(n-s) p} S_{\mu, s}^{\frac{n-s}{p-s}}(\operatorname{by}(3.10)),\end{cases}
\end{aligned}
$$

which completes the proof of Theorem 1.2.

Proof of Theorem 1.3 Since $S_{0}=S, \lim _{\mu \rightarrow \bar{\mu}} S_{\mu}=0$ and $S_{\mu}$ is continuous with respect to $\mu$, we deduce that there exists $\mu^{*} \in(0, \bar{\mu})$ such that $\frac{1}{2} S^{\frac{n}{p}} \leq S_{\mu}^{\frac{n}{p}}$ for $0<\mu \leq \mu^{*}$ and $\frac{1}{2} S^{\frac{n}{p}}>S_{\mu}^{\frac{n}{p}}$ for $\mu^{*}<\mu<\bar{\mu}$. From this fact, we can define $\mu^{*}$ as above.

(1) By Proposition 3.2 and the definition of $\mu^{*}$, it suffices to prove

$$
c_{\mu}<\frac{1}{2 n} S^{\frac{n}{p}} .
$$

Let $B(x, r)$ be a ball containing $\Omega, \partial B(x, r) \cap \partial \Omega \neq \emptyset, x_{0} \in \partial B(x, r) \cap \partial \Omega$. Then without loss of generality we may suppose that $\Omega \subset\left\{x \in \mathbb{R}^{n}, x_{n}>x_{0}^{n}\right\}$, where $x_{0}=\left(x_{1}^{0}, x_{2}^{0}, \ldots, x_{n}^{0}\right)$. Since $\mu>0, \eta>0$, we have

$$
\max _{t>0} F_{\mu}\left(t U_{x_{0}}^{\varepsilon}\right) \leq y_{\varepsilon}:=\max _{t>0}\left\{\frac{t^{p}}{p} \int_{\Omega}\left(\left|\nabla U_{x_{0}}^{\varepsilon}\right|^{p}+\lambda\left|U_{x_{0}}^{\varepsilon}\right|^{p}\right) d x-\frac{t^{p^{*}}}{p^{*}} \int_{\Omega}\left|U_{x_{0}}^{\varepsilon}\right|^{p^{*}} d x\right\},
$$

and by Lemma 3.4 in [21], we have

$$
y_{\varepsilon}<\frac{1}{2 n} S^{n / p} .
$$

It follows from the definition of $c_{\mu}$ and (3.16) that (3.15) holds.

(2) For the case that $\mu^{*}<\mu<\bar{\mu}$, let $v_{\varepsilon}$ and $t_{\varepsilon}$ be defined as in the proof of Theorem 1.2. Since $\mu>0, \eta>0$, we have

$$
\begin{aligned}
\max _{t>0} F_{\mu}\left(t v_{\varepsilon}\right)= & F_{\mu}\left(t_{\varepsilon} v_{\varepsilon}\right) \\
\leq & \max _{t>0}\left\{\frac{t^{p}}{p} \int_{\Omega}\left(\left|\nabla v_{\varepsilon}\right|^{p}-\mu \frac{\left|v_{\varepsilon}\right|^{p}}{|x|^{p}}\right) d x-\frac{t^{p^{*}}}{p^{*}} \int_{\Omega}\left|v_{\varepsilon}\right|^{p^{*}} d x\right\} \\
& +\frac{t_{\varepsilon}^{p}}{p} \int_{\Omega} \lambda\left|v_{\varepsilon}\right|^{p} d x-\eta \frac{t_{\varepsilon}^{q}}{q} \int_{\Omega}\left|v_{\varepsilon}\right|^{q} d x
\end{aligned}
$$




$$
\begin{aligned}
\leq & \max _{t>0}\left\{\frac{t^{p}}{p} \int_{\Omega}\left|\nabla v_{\varepsilon}\right|^{p} d x-\frac{t^{p^{*}}}{p^{*}} \int_{\Omega}\left|v_{\varepsilon}\right|^{p^{*}} d x\right\} \\
& +\frac{t_{\varepsilon}^{p}}{p} \int_{\Omega} \lambda\left|v_{\varepsilon}\right|^{p} d x-\eta \frac{t_{\varepsilon}^{q}}{q} \int_{\Omega}\left|v_{\varepsilon}\right|^{q} d x .
\end{aligned}
$$

By a similar argument as in the proof of (3.5) for the special case $s=0, \mu=0$, we have

$$
c_{\mu}<\frac{1}{n} S^{\frac{n}{p}}
$$

The proof of Theorem 1.3 is complete.

\section{Appendix}

In this appendix, we give some lemmas and detailed proofs for the convenience of the reader. In the following, assume that $\Omega \subset \mathbb{R}^{n}$ is a bounded domain and $\partial \Omega \in C^{1}$.

\section{Lemma A.1 Define}

$$
\lambda_{1}=\inf \left\{\int_{\Omega}\left(|\nabla u|^{p}-\mu \frac{|u|^{p}}{|x|^{p}}\right) d x+\int_{\partial \Omega} \alpha(x)|u|^{p} d \sigma ; \int_{\Omega}|u|^{p} d x=1, u \in W^{1, p}(\Omega)\right\}
$$

then $\lambda_{1}$ is obtained.

Proof Let $\left\{u_{m}\right\}$ be the minimizing sequence for $\lambda_{1}$. That is,

$$
\lim _{m \rightarrow+\infty} \int_{\Omega}\left(\left|\nabla u_{m}\right|^{p}-\mu \frac{\left|u_{m}\right|^{p}}{|x|^{p}}\right) d x+\int_{\partial \Omega} \alpha(x)\left|u_{m}\right|^{p} d x=\lambda_{1}, \quad \int_{\Omega}\left|u_{m}\right|^{p} d x=1
$$

By the Sobolev-Hardy inequality, and $\mu \leq \bar{\mu}, \alpha(x) \geq 0$, we have

$$
\int_{\Omega}\left(\left|\nabla u_{m}\right|^{p}-\mu \frac{\left|u_{m}\right|^{p}}{|x|^{p}}\right) d x+\int_{\partial \Omega} \alpha(x)\left|u_{m}\right|^{p} d x \geq\left(1-\frac{\mu}{\bar{\mu}}\right) \int_{\Omega}\left|\nabla u_{m}\right|^{p} d x \geq 0
$$

Then $u_{m}$ is bounded in $W^{1, p}(\Omega)$, there exists $u \in W^{1, p}(\Omega)$ such that, up to a subsequence still denoted by $u_{m}$,

$$
u_{m} \rightarrow u \text { weakly in } W^{1, p}(\Omega) \text { as } m \rightarrow+\infty \text {. }
$$

By the Sobolev imbedding theorem we have

$$
\begin{aligned}
& u_{m} \rightarrow u \text { in } L^{p}(\Omega) \text { and } L^{p}(\partial \Omega) \text { as } m \rightarrow+\infty, \\
& u_{m} \rightarrow u \text { a.e. in } \Omega \text { as } m \rightarrow+\infty .
\end{aligned}
$$

Thus by the Fatou lemma we have

$$
\begin{aligned}
& \int_{\Omega}\left(|\nabla u|^{p}-\mu \frac{|u|^{p}}{|x|^{p}}\right) d x+\int_{\partial \Omega} \alpha(x)|u|^{p} d x \\
& \leq \lim _{m \rightarrow+\infty}\left[\int_{\Omega}\left(\left|\nabla u_{m}\right|^{p}-\mu \frac{\left|u_{m}\right|^{p}}{|x|^{p}}\right) d x+\int_{\partial \Omega} \alpha(x)\left|u_{m}\right|^{p} d x\right] .
\end{aligned}
$$


And since $\lim _{m \rightarrow \infty} \int_{\Omega}\left|u_{m}\right|^{p} d x=\int_{\Omega}|u|^{p} d x$, from (A.1) (A.2), the proof of the lemma is complete.

Lemma A.2 For any $\delta>0$, there exists a constant $C=C(\delta)>0$ such that

$$
\int_{\Omega} \frac{|u|^{p}}{|x|^{p}} d x \leq\left(\frac{1}{\bar{\mu}}+\delta\right) \int_{\Omega}|\nabla u|^{p} d x+C(\delta) \int_{\Omega}|u|^{p} d x
$$

for $u \in W^{1, p}(\Omega)$.

Proof The proof is similar to that in [12]. Here for convenience we give the details of the proof. For $y \in \mathbb{R}^{n}$, denote the unit ball centered at $y$ by $B_{1}(y)$ and domain

$$
D=B_{1}(y) \cap\left\{x_{n}>h\left(x^{\prime}\right)\right\}
$$

where $h\left(x^{\prime}\right)$ is a $C^{1}$ function defined in $\left\{x^{\prime} \in \mathbb{R}^{n-1}:\left|x^{\prime}-y^{\prime}\right|<1\right\}$ with $y_{n}=h\left(y_{1}, \ldots, y_{n-1}\right)$ and $\nabla h$ vanishing at $y^{\prime}=\left(y_{1}, \ldots, y_{n-1}\right), h \geq 0$. Employing similar arguments in Lemma 2.1 of [22], it can be proved that if $u \in W^{1, p}(D)$ with $\operatorname{supp} u \in B_{1}(y)$, then $\forall \varepsilon>0$, there exists a constant $r>0$ depending on $\varepsilon$ such that

$$
\int_{D} \frac{|u|^{p}}{|x|^{p}} d x \leq\left(\frac{1}{\bar{\mu}}+\varepsilon\right) \int_{D}|\nabla u|^{p} d x
$$

provided $|\nabla h| \leq r$. In fact, if $h \equiv 0$,

$$
\int_{D}|\nabla u|^{p} d x=\frac{1}{2} \int_{B_{1}(y)}|\nabla u|^{p} d x \geq \frac{\bar{\mu}}{2} \int_{B_{1}(y)} \frac{|u|^{p}}{|x|^{p}} d x=\bar{\mu} \int_{D} \frac{|u|^{p}}{|x|^{p}} d x
$$

If $h \geq 0, h \neq \equiv$, make the coordinate transformation

$$
z^{\prime}=x^{\prime}, \quad z_{n}=x_{n}-h\left(x^{\prime}\right)
$$

which straightens the bottom of $D$, and write $z=F(x)$, then

$$
\begin{aligned}
& \partial_{z_{i}} u(x)=\partial_{x_{i}} u(x)+\partial_{x_{n}} u(x) \partial_{x_{i}} h\left(x^{\prime}\right), \quad i=1,2, n-1, \\
& \left|\partial_{z_{i}} u(x)\right|^{2}=\left|\partial_{x_{i}} u(x)\right|^{2}+\left|\partial_{x_{n}} u(x) \partial_{x_{i}} h\left(x^{\prime}\right)\right|^{2}+2\left|\partial_{x_{n}} u(x) \partial_{x_{i}} u(x) \partial_{x_{i}} h\left(x^{\prime}\right)\right|^{2} \\
& \left|\nabla_{z} u(x)\right|^{2} \leq\left|\nabla_{x} u(x)\right|^{2}+2|\nabla h|^{2}\left|\nabla_{x} u(x)\right|^{2} \\
& |z| \leq|x|
\end{aligned}
$$

Denote $D_{1}=F(D)$, then we have

$$
\begin{aligned}
\int_{D}|\nabla u|^{2} d x & \geq\left(1-2|\nabla h|^{2}\right) \int_{D_{1}}\left|\nabla_{z} u\right|^{2} d z \\
& \geq\left(1-2|\nabla h|^{2}\right) \bar{\mu} \int_{D_{1}} \frac{|u|^{p}}{|z|^{p}} d z \geq\left(1-2|\nabla h|^{2}\right) \bar{\mu} \int_{D} \frac{|u|^{p}}{|x|^{p}} d x
\end{aligned}
$$

Then (A.3) is obtained provided $|\nabla h| \leq r$. 
Let $\varepsilon$ be a small positive constant to be determined later, and let $\left(\varphi_{k}\right)_{k=1}^{m}$ be a partition of unity on $\bar{\Omega}$ with $\operatorname{diam}\left(\operatorname{supp} \varphi_{k}\right) \leq r$ for each $k$, where $\operatorname{diam}\left(\operatorname{supp} \varphi_{k}\right)$ is the diameter of the domain $\operatorname{supp} \varphi_{k}$. From (A.3), we see

$$
\int_{\Omega} \frac{\left|\varphi_{k} u\right|^{p}}{|x|^{p}} d x \leq\left(\frac{1}{\bar{\mu}}+\varepsilon\right) \int_{\Omega}\left|\nabla\left(\varphi_{k} u\right)\right|^{p} d x, \quad \forall 1 \leq k \leq m, u \in W^{1, p}(\Omega)
$$

for sufficiently small $r$. Hence

$$
\begin{aligned}
\int_{\Omega} \frac{|u|^{p}}{|x|^{p}} d x & \leq \int_{\Omega} \sum_{k=1}^{m} \varphi_{k} \frac{|u|^{p}}{|x|^{p}} d x \leq\left(\frac{1}{\bar{\mu}}+\varepsilon\right) \sum_{k=1}^{m} \int_{\Omega}\left|\nabla\left(\varphi_{k}^{\frac{1}{p}} u\right)\right|^{p} d x \\
& \leq\left(\frac{1}{\bar{\mu}}+\varepsilon\right) \sum_{k=1}^{m} \int_{\Omega} \varphi_{k}\left(|\nabla u|^{p}+C \sum_{j=1}^{p}|\nabla u|^{p-j}+C|u|^{p}\right) d x \\
& \leq\left(\frac{1}{\bar{\mu}}+\varepsilon\right)\left[(1+\varepsilon) \int_{\Omega}|\nabla u|^{p} d x+C(\varepsilon) \int_{\Omega}|u|^{p} d x\right] .
\end{aligned}
$$

As a consequence, by choosing $\varepsilon$ appropriately, we obtain the desired result.

Lemma A.3 For $\lambda>-\lambda_{1}$, the norm

$$
\|u\|=\left[\int_{\Omega}\left(|\nabla u|^{p}-\mu \frac{|u|^{p}}{|x|^{p}}+\lambda|u|^{p}\right) d x+\int_{\partial \Omega} \alpha(x)|u|^{p} d \sigma\right]^{\frac{1}{p}}
$$

is equivalent to $\|\cdot\|_{W^{1, p}(\Omega)}$.

Proof For simplicity, we suppose $\alpha(x) \equiv 0$. We only consider the case $0<\mu<\bar{\mu}$ since the case $\mu \leq 0$ is similar.

First we have

$$
\int_{\Omega}\left(|\nabla u|^{p}-\mu \frac{|u|^{p}}{|x|^{p}}+\lambda|u|^{p}\right) d x \geq\left(\lambda+\lambda_{1}\right) \int_{\Omega}|u|^{p} d x, \quad \forall u \in W^{1, p}(\Omega) .
$$

By Lemma A.2, we deduce that for all $u \in W^{1, p}(\Omega)$,

$$
\begin{aligned}
\frac{C(\delta) \mu}{\lambda+\lambda_{1}} \int_{\Omega}\left(|\nabla u|^{p}-\mu \frac{|u|^{p}}{|x|^{p}}+\lambda|u|^{p}\right) d x & \geq C(\delta) \mu \int_{\Omega}|u|^{p} d x \\
& \geq \mu \int_{\Omega} \frac{|u|^{p}}{|x|^{p}} d x-\mu\left(\frac{1}{\bar{\mu}}+\delta\right) \int_{\Omega}|\nabla u|^{p} d x .
\end{aligned}
$$

Hence, for $\delta>0$ small enough,

$$
\begin{aligned}
(1 & \left.+\frac{C(\delta) \mu}{\lambda+\lambda_{1}}\right) \int_{\Omega}\left(|\nabla u|^{p}-\mu \frac{|u|^{p}}{|x|^{p}}+\lambda|u|^{p}\right) d x \\
& \geq\left[1-\mu\left(\frac{1}{\bar{\mu}}+\delta\right)\right] \int_{\Omega}|\nabla u|^{p} d x+\lambda \int_{\Omega}|u|^{p} d x \\
& \geq c \int_{\Omega}|\nabla u|^{p} d x+c \int_{\Omega}|u|^{p} d x,
\end{aligned}
$$


which implies that

$$
\|u\| \geq c\|u\|_{W^{1, p}(\Omega)}
$$

for some $c>0$.

On the other hand, it is easy to check that

$$
\|u\| \leq C\|u\|_{W^{1, p}(\Omega)}
$$

for some $C>0$. As a result, we complete the proof.

Lemma A.4 Let $\left\{u_{m}\right\}_{m}$ be a Palais-Smale sequence for $F_{\mu}(u)$ at level $d \in \mathbb{R}$. Then $\left\{u_{m}\right\}_{m}$ is bounded in $W^{1, p}(\Omega)$. Moreover, every Palais-Smale sequence for $F_{\mu}(u)$ at a level zero converges strongly to zero.

Proof Since $\left\{u_{m}\right\}_{m}$ is a Palais-Smale sequence for $F_{\mu}(u)$ at level $d \in \mathbb{R}$, we have

$$
\begin{aligned}
d+o(1) & =F_{\mu}\left(u_{m}\right)-\frac{1}{p}\left\langle F_{\mu}^{\prime}\left(u_{m}\right), u_{m}\right\rangle \\
& =\left(\frac{1}{p}-\frac{1}{p^{*}(s)}\right) \int_{\Omega} \frac{\left|u_{m}\right|^{p^{*}(s)}}{|x|^{s}} d x+\left(\frac{1}{p}-\frac{1}{q}\right) \int_{\Omega}\left|u_{m}\right|^{q} d x .
\end{aligned}
$$

Hence

$$
\int_{\Omega} \frac{\left|u_{m}\right|^{p^{*}(s)}}{|x|^{s}} d x \leq C, \quad \int_{\Omega}\left|u_{m}\right|^{q} d x \leq C
$$

since $q, p^{*}(s)>p$.

As a result, by Lemma A.3,

$$
\left\|u_{m}\right\|_{W^{1, p}(\Omega)}^{p} \leq c\left\|u_{m}\right\|^{p}=p c d+\frac{p c}{p^{*}(s)} \int_{\Omega} \frac{\left|u_{m}\right|^{p^{*}(s)}}{|x|^{s}} d x+\frac{p c}{q} \int_{\Omega}\left|u_{m}\right|^{q} d x+o(1) \leq C .
$$

Take $d=0$, from $(\mathrm{A} .7)$ then

$$
\int_{\Omega} \frac{\left|u_{m}\right|^{p^{*}(s)}}{|x|^{s}} d x \rightarrow 0, \quad \int_{\Omega}\left|u_{m}\right|^{q} d x \rightarrow 0, \quad \text { as } m \rightarrow+\infty
$$

and from (A.8), we have $\left\|u_{m}\right\|_{W^{1, p}(\Omega)}^{p} \rightarrow 0$, the lemma is complete.

Let $\left\{u_{m}\right\}_{m}$ be a Palais-Smale sequence of $F_{\mu}(u)$, we shall assume that, up to a subsequence,

$$
u_{m} \rightarrow u_{0} \text { weakly in } W^{1, p}(\Omega) \text { as } m \rightarrow+\infty \text {. }
$$

Then we have the following lemma.

Lemma A.5 $D F_{\mu}\left(u_{0}\right)=0$. 
Proof We have to prove that $\left\langle v, D F_{\mu}\left(u_{0}\right)\right\rangle=0$ for every $v \in W^{1, p}(\Omega)$ as $m \rightarrow+\infty$. Since $\partial \Omega \in C^{1}$, it is enough to prove that the above relation holds for every restriction to $\Omega$ of a $C_{0}^{\infty}\left(\mathbb{R}^{n}\right)$ function $\phi$.

From (A.9), the Sobolev imbedding theorem and Lemma 3.2(2) in [20], we have as $m \rightarrow+\infty$

$$
\begin{aligned}
& \nabla u_{m} \rightarrow \nabla u_{0} \text { weakly in } L^{p}(\Omega), \\
& u_{m} \rightarrow u_{0} \text { in } L^{p^{*}(s)-1}\left(\Omega,|x|^{-s}\right), \\
& u_{m} \rightarrow u_{0} \text { in } L^{p-1}\left(\Omega,|x|^{-p}\right), \\
& u_{m} \rightarrow u_{0} \text { in } L^{p-1}(\partial \Omega), \\
& u_{m} \rightarrow u_{0} \text { in } L^{q}(\Omega) \text { for } 1<q<p^{*},
\end{aligned}
$$

then

$$
\begin{aligned}
\left\langle\phi, D F_{\mu}\left(u_{m}\right)\right\rangle= & \int_{\Omega}\left(\left|\nabla u_{m}\right|^{p-2} \nabla u_{m} \nabla \phi-\mu \frac{u_{m}\left|u_{m}\right|^{p-2} \phi}{|x|^{p}}\right) d x-\int_{\Omega} \frac{u_{m}\left|u_{m}\right|^{p^{*}(s)-2} \phi}{|x|^{s}} d x \\
& -\eta \int_{\Omega}\left|u_{m}\right|^{q-2} u_{m} \phi d x+\lambda \int_{\Omega}\left|u_{m}\right|^{p-2} u_{m} \phi d x+\int_{\partial \Omega} \alpha(x)\left|u_{m}\right|^{p-2} u_{m} \phi d x \\
\rightarrow & \left\langle\phi, D F_{\mu}\left(u_{0}\right)\right\rangle \text { as } m \rightarrow+\infty,
\end{aligned}
$$

i.e.,

$$
0=\lim _{m \rightarrow+\infty}\left\langle\phi, D F_{\mu}\left(u_{m}\right)\right\rangle=\left\langle\phi, D F_{\mu}\left(u_{0}\right)\right\rangle
$$

Put $y_{m}=u_{m}-u_{0}$, then $y_{m} \rightarrow 0$ weakly in $W^{1, p}(\Omega)$. Then we have the following lemma.

Lemma A.6 $\left\{y_{m}\right\}_{m}$ is a Palais-Smale sequence for $F_{\mu}(u)$ at level $d_{0}=d-F_{\mu}\left(u_{0}\right)$.

Proof Since $u_{m}$ is bounded in $W^{1, p}(\Omega)$, by the Sobolev-Hardy inequality $\int_{\Omega} \frac{\left|u_{m}\right|^{p^{*}(s)}}{|x|^{s}} d x$, $\int_{\Omega} \frac{\left|u_{m}\right|^{p}}{|x|^{p}} d x$ is bounded. That is, $u_{m}$ is bounded in $L^{p^{*}(s)}\left(\Omega,|x|^{-s}\right), L^{p}\left(\Omega,|x|^{-p}\right)$. And as $m \rightarrow+\infty$

$$
\begin{aligned}
& u_{m} \rightarrow u_{0} \text { weakly in } W^{1, p}(\Omega), \\
& u_{m} \rightarrow u_{0} \text { in } L^{p}(\Omega) \\
& u_{m} \rightarrow u \text { a.e. in } \Omega .
\end{aligned}
$$

By the Brezis and Lieb lemma [9] we obtain, as $m \rightarrow+\infty$,

$$
\begin{aligned}
& \int_{\Omega} \frac{\left|y_{m}\right|^{p^{*}(s)}}{|x|^{s}} d x=\int_{\Omega} \frac{\left|u_{m}\right|^{p^{*}(s)}}{|x|^{s}} d x-\int_{\Omega} \frac{\left|u_{0}\right|^{p^{*}(s)}}{|x|^{s}} d x+o(1), \\
& \int_{\Omega} \frac{\left|y_{m}\right|^{p}}{|x|^{p}} d x=\int_{\Omega} \frac{\left|u_{m}\right|^{p}}{|x|^{p}} d x-\int_{\Omega} \frac{\left|u_{0}\right|^{p}}{|x|^{p}} d x+o(1) .
\end{aligned}
$$


Similarly,

$$
\begin{aligned}
& \int_{\Omega}\left|\nabla y_{m}\right|^{p} d x=\int_{\Omega}\left|\nabla u_{m}\right|^{p} d x-\int_{\Omega}\left|\nabla u_{0}\right|^{p} d x+o(1), \\
& \int_{\Omega}\left|y_{m}\right|^{q} d x=\int_{\Omega}\left|u_{m}\right|^{q} d x-\int_{\Omega}\left|u_{0}\right|^{q} d x+o(1), \quad \forall p \leq q \leq p^{*}(s), \\
& \int_{\partial \Omega}\left|y_{m}\right|^{p} d \sigma=\int_{\partial \Omega}\left|u_{m}\right|^{p} d \sigma-\int_{\partial \Omega}\left|u_{0}\right|^{p} d \sigma+o(1) .
\end{aligned}
$$

From (A.10)-(A.14), we obtain $F_{\mu}\left(y_{m}\right)=F_{\mu}\left(u_{m}\right)-F_{\mu}\left(u_{0}\right)+o(1)=d-F_{\mu}\left(u_{0}\right)+o(1)$. On the other hand, for any test function $v \in W^{1, p}(\Omega)$,

$$
\begin{aligned}
& \int_{\Omega} \frac{\left|y_{m}\right|^{p^{*}(s)-2} y_{m} v}{|x|^{s}} d x=\int_{\Omega} \frac{\left|u_{m}\right|^{p^{*}(s)-2} u_{m} v}{|x|^{s}} d x-\int_{\Omega} \frac{\left|u_{0}\right|^{p^{*}(s)-2} u_{0} v}{|x|^{s}} d x+o(1), \\
& \int_{\Omega}\left|y_{m}\right|^{q-2} y_{m} v d x=\int_{\Omega}\left|u_{m}\right|^{q-2} u_{m} v d x-\int_{\Omega}\left|u_{0}\right|^{q} u_{0} v d x+o(1), \quad \forall p \leq q<p^{*}(s), \\
& \int_{\partial \Omega}\left|y_{m}\right|^{p-2} y_{m} v d \sigma=\int_{\partial \Omega}\left|u_{m}\right|^{p-2} u_{m} v d \sigma-\int_{\partial \Omega}\left|u_{0}\right|^{p-2} u_{0} v d \sigma+o(1), \\
& \int_{\Omega} \frac{\left|y_{m}\right|^{p-2} y_{m} v}{|x|^{p}} d x=\int_{\Omega} \frac{\left|u_{m}\right|^{p-2} u_{m} v}{|x|^{p}} d x-\int_{\Omega} \frac{\left|u_{0}\right|^{p-2} u_{0} v}{|x|^{p}} d x+o(1), \\
& \int_{\Omega}\left|\nabla y_{m}\right|^{p-2} \nabla y_{m} \nabla v d x=\int_{\Omega}\left|\nabla u_{m}\right|^{p-2} \nabla u_{m} \nabla v d x-\int_{\Omega}\left|\nabla u_{0}\right|^{p-2} \nabla u_{0} \nabla v d x+o(1),
\end{aligned}
$$

that is, $\left\langle v, D F_{\mu}\left(y_{m}\right)\right\rangle=\left\langle v, D F_{\mu}\left(u_{m}\right)\right\rangle-\left\langle v, D F_{\mu}\left(u_{0}\right)\right\rangle=o(1)$, thus we complete the proof of the lemma.

\section{Competing interests}

The authors declare that they have no competing interests.

\section{Authors' contributions}

All authors typed, read and approved the final manuscript.

\section{Acknowledgements}

The authors are grateful to the referees for many valuable suggestions to make the paper more readable. Research was supported by the Natural Science Foundation of China, No. 11101160 and No. 11271141.

\section{Received: 22 May 2015 Accepted: 24 September 2015 Published online: 09 October 2015}

\section{References}

1. Caffarelli, L, Kohn, R, Nirenberg, L: First order interpolation inequalities with weights. Compos. Math. 53, 259-275 (1984)

2. Garcia Azorero, JP, Peral Alonso, I: Hardy inequalities and some critical elliptic and parabolic problems. J. Differ. Equ. 144, 441-476 (1998). doi:10.1006/jdeq.1997.3375

3. Adimurthi, YSL: Critical Sobolev exponent problem in $\mathbb{R}^{N}(N \geq 4)$ with Neumann boundary condition. Proc. Indian Acad. Sci. Math. Sci. 100, 275-284 (1990). doi:10.1007/BF02837850

4. Cao, DM, Peng, SJ: A global compactness result for singular elliptic problems involving critical Sobolev exponent. Proc. Am. Math. Soc. 131, 1857-1866 (2003). doi:10.1090/S0002-9939-02-06729-1

5. Cao, DM, Peng, SJ: A note of the sign-changing solutions to elliptic problem with critical Sobolev and Hardy terms. J. Differ. Equ. 193, 424-434 (2003). doi:10.1016/50022-0396(03)00118-9

6. Chabrowski, J: On the nonlinear Neumann problem involving the critical Sobolev exponent and Hardy potential. Rev. Mat. Complut. 17, 195-227 (2004). doi:10.5209/rev-REMA.2004.v17.n1.16800

7. Jannelli, E: The role played by space dimension in elliptic critical problems. J. Differ. Equ. 156, 407-426 (1999). doi:10.1006/jdeq.1998.3589

8. Smets, D: Nonlinear Schrödinger equations with Hardy potential and critical nonlinearities. Trans. Am. Math. Soc. 357, 2909-2938 (2005). doi:10.1090/50002-9947-04-03769-9

9. Brezis, $\mathrm{H}$, Lieb, E: A relation between pointwise convergence of functions and convergence of functionals. Proc. Am. Math. Soc. 88, 486-490 (1983). doi:10.2307/2044999 
10. Struwe, M: A global compactness result for elliptic boundary value problems involving limiting nonlinearities. Math. Z. 187, 511-517 (1984). doi:10.1007/BF01174186

11. Pierrotti, D, Terracini, S: On a Neumann problem with critical exponent and critical nonlinearity on the boundary. Commun. Partial Differ. Equ. 20, 1155-1187 (1995). doi:10.1080/03605309508821128

12. Deng, YB, Jin, LY, Peng, SJ: A Robin boundary problem with Hardy potential and critical nonlinearities. J. Anal. Math. 104, 125-154 (2008). doi:10.1007/s11854-008-0019-3

13. Li, YY, Guo, QQ, Niu, PC: Global compactness results for quasilinear elliptic problems with combined critical Sobolev-Hardy terms. Nonlinear Anal. 74, 1445-1464 (2011). doi:10.1016/..na.2010.10.018

14. Deng, YB, Jin, LY, Peng, SJ: Solutions of Schrödinger equations with inverse square potential and critical nonlinearity. J. Differ. Equ. 253, 1376-1398 (2012). doi:10.1016/j.jde.2012.05.009

15. Jin, LY, Deng, YB: A global compact result for a semilinear elliptic problem with Hardy potential and critical nonlinearities on $\mathbb{R}^{n}$. Sci. China Ser. A 53(2), 385-400 (2010). doi:10.1007/s11425-009-0075-x

16. Aubin, T: Problèmes isopérimétriques et espaces de Sobolev, C. R. Acad. Sci. Paris Sér. A-B 280, 279-281 (1975)

17. Kang, DS: On the quasilinear elliptic problem with a critical Hardy-Sobolev exponent and a Hardy term. Nonlinear Anal. 69, 2432-2444 (2008). doi:10.1016/j.na.2007.08.022

18. Brezis, H, Nirenberg, L: Positive solutions of nonlinear elliptic equations involving critical exponents. Commun. Pure Appl. Math. 36, 437-477 (1983). doi:10.1002/cpa.3160360405

19. Lindqvist, P: Notes on the $p$-Laplace equation. Report. University of Jyväskylä, Department of Mathematics and Statistics, 102. University of Jyväskylä, Jyväskylä (2006)

20. Ghoussoub, N, Yuan, C: Multiple solutions for quasi-linear PDEs involving the critical Sobolev and Hardy exponents. Trans. Am. Math. Soc. 352, 5703-5743 (2000). doi:10.1090/S0002-9947-00-02560-5

21. Abreu, EAM, do Ó, JM, Medeiros, ES: Multiplicity of positive solutions for a class of quasilinear nonhomogeneous Neumann problems. Nonlinear Anal. 60, 1443-1471 (2005). doi:10.1016/j.na.2004.09.058

22. Wang, XJ: Neumann problem for semilinear elliptic equations involving critical Sobolev exponents. J. Differ. Equ. 93 , 283-310 (1991). doi:10.1016/0022-0396(91)90014-Z

\section{Submit your manuscript to a SpringerOpen ${ }^{\circ}$ journal and benefit from:}

- Convenient online submission

Rigorous peer review

- Immediate publication on acceptance

- Open access: articles freely available online

- High visibility within the field

- Retaining the copyright to your article 